\title{
Anchor Free IP Mobility
}

\author{
Mohammed Al-Khalidi, Nikolaos Thomos, Martin J. Reed, Mays F. AL-Naday and Dirk Trossen
}

\begin{abstract}
Efficient mobility management techniques are critical in providing seamless connectivity and session continuity between a mobile node and the network during its movement. However, current mobility management solutions generally require a central entity in the network core, tracking IP address movement and anchoring traffic from source to destination through point-to-point tunnels. Intuitively, this approach suffers from scalability limitations as it creates bottlenecks in the network, due to sub-optimal routing via the anchor point. This is often termed "dog-leg" routing. Meanwhile, alternative anchorless, solutions are not feasible due to the current limitations of the IP semantics, which strongly tie addressing information to location. In contrast, this paper introduces a novel anchorless mobility solution that overcomes these limitations by exploiting a new path-based forwarding fabric together with emerging mechanisms from information-centric networking. These mechanisms decouple the end-system IP address from the path based data forwarding to eliminate the need for anchoring traffic through the network core; thereby, allowing flexible path calculation and service provisioning. Furthermore, by eliminating the limitation of routing via the anchor point, our approach reduces the network cost compared to anchored solutions through bandwidth saving while maintaining comparable handover delay. The proposed solution is applicable to both cellular and large-scale wireless LAN networks that aim to support seamless handover in a single operator domain scenario. The solution is modeled as a Markov-chain which applies a topological basis to describe mobility. The validity of the proposed Markovian model was verified through simulation of both random walk mobility on random geometric networks and trace information from a large-scale, city wide data set. Evaluation results illustrate a significant reduction in the total network traffic cost by $45 \%$ or more when using the proposed solution, compared to Proxy Mobile IPv6.
\end{abstract}

Index Terms-IP-over-ICN, Mobile IP, Proxy MIPv6, LTE, GPRS, Handover.

\section{INTRODUCTION}

$\mathrm{T}$ HE significant progress achieved in mobile technologies, allowing users to enjoy Internet services during movement, relies on mobility management protocols. Mobility management is a challenging research topic since it largely affects users' experience in respect of preventing frequent disconnections and ensuring session continuity [1]. This is particularly critical in the emerging form of mobile networking, which focuses on providing IP-based services over well managed wireless networks. Typically, such networks have a wide coverage with a known topology map; designed and maintained by a single operator, as in current cellular networks.

Network-based mobility management is a desirable approach from an operator's perspective, in such well defined networks. This is because it allows service providers to enable mobility support without any user interaction or mobile node (MN) modification [2] [3]. However, achieving efficient mobility management in existing IP networks has been a challenge, because the IP architecture ties the end host address to its location; thereby, a moving host cannot naturally maintain a single identifier when attaching to different points in the network. This has arisen as a critical hinder for supporting mobility features, such as seamless handover and session continuity.

- Mohammed Al-Khalidi, Nikolaos Thomos, Martin J. Reed and Mays F. AL-Naday are with the School of Computer Science and Electronic Engineering, University of Essex, Colchester, CO4 3SQ, UK.

E-mails: (mshawk,nthomos,mjreed,mfhaln)@essex.ac.uk

- Dirk Trossen is with InterDigital Europe Ltd., London, EC2A 3QR, UK. Email: Dirk.Trossen@InterDigital.com

This work was carried out within the project POINT, which has received funding from the European Union's Horizon 2020 research and innovation programme under grant agreement No 643990.
The problem has generally been circumvented by using IP tunnelling through a central anchor point, which tracks the IP addresses of moving hosts and instructs the access gateways to provide the same IP address to the same host. This form of the so-called "dog-leg" routing is shown in Fig. 1. This approach introduces tunnelling overhead and sub-optimal routing with an overall increase in traffic and latency. Such problems are particularly evident with propositions to increasingly localize traffic delivery, e.g., when accessing locally available content or user generated multicast/broadcast traffic. The motivation of this paper is to overcome the problem caused by anchoring through recent work that utilizes path-based routing that is not directly linked to end-point identifiers.

An example of the anchor-based approach is the Proxy Mobile IPv6 (PMIPv6) solution [4]; standardised by the Internet Engineering Task Force (IETF). In PMIPv6, a central Local Mobility Anchor (LMA) is responsible for maintaining reachability to the MN's IP address during the latter's movement between Mobile Access Gateways (MAGs) in the PMIPv6 domain. The LMA updates the address binding cache in a binding table and maintains a tunnel to the MN's MAG for packet delivery. The MAG is responsible for detecting the MN's movement and initiating binding registration on behalf of the MN [5] [6]. PMIPv6 also supports an IPv4 stack and dual stack mobility modes [7]. Alternative anchor-based solutions have also been adapted by 3GPP in the Global System for Mobile Communications (GSM), Universal Mobile Telecommunications System (UMTS) and Long-Term Evolution (LTE) core networks. An overview of these solutions is provided in Section 5. Notably, the solutions described here are designed to function in a single operator domain and likewise, this paper, focuses on a 


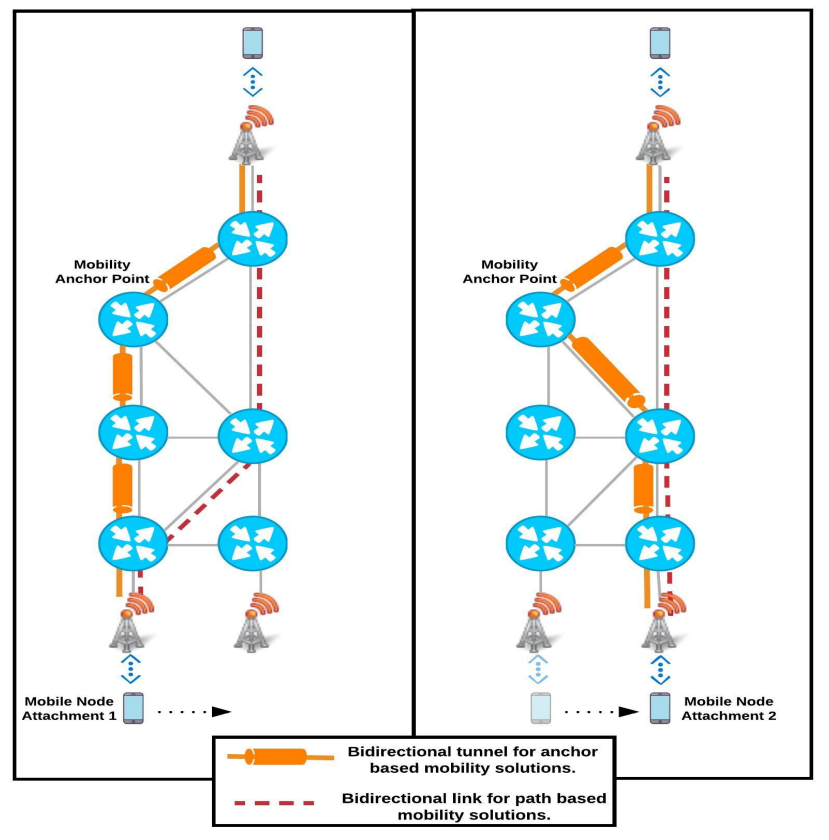

Fig. 1: Packet Delivery Routes in Anchor Based vs. Path Based Mobility Solutions.

single operator solution. Inter-domain mobility solutions are out of the scope of this paper.

Noticeably, PMIPv6 (as with other anchor-based solutions) suffers from a number of drawbacks; first of all, it violates network end-to-end transparency: although it provides user experience transparency, an essential goal for mobility support, it does not provide network addressing transparency which requires unaltered mechanisms for the flow of packets and unaltered logical addressing between source and destination [8]. Secondly, PMIPv6 increases network fragility due to the explosive growth of the tracking state in the LMA for all MNs in the domain. Thirdly, it imposes processing complexity in the network core (LMA) and edges (MAGs) to support the necessary protocol functionality during mobility [9] [10].

The path based approach in this paper uses new forwarding architectures that rely purely on path information for the end-to-end forwarding of packets instead of relying on host address-based communication. Solutions such as LIPSIN [11] [12] and BIER [13] utilize path information stored in the forwarded packet to deliver a packet traversing through the network. In these alternative path based approaches, the route computation determines an end-toend path that is encoded into the packet header while the forwarding operation is considerably simpler than IP forwarding by virtue of executing a simple set membership test which can be efficiently implemented. Recent advances have shown that these path based approaches can be carried out in commercially available SDN switches with a switching table size that is constant and considerably lower than traditional end-host address-based solutions [12]. Mobility management in these architectures simply requires (partial) recomputation of a path with the opportunity to deliver the data over an optimal path after every handover operation as described in Section 2.

To support a path-based mobility solution, as with ex- isting IP mobility, a control plane is required. In common with the comparison systems (3GPP and PMIPv6), this work uses a centralized control-plane. The investigation of a new control plane is out of the scope of this paper, thus, we base our proposal on an existing solution; namely Information Centric Networking (ICN) [14] [15], specifically that developed in PURSUIT [15]. PURSUIT employs a PublishSubscribe paradigm for a path-based information dissemination that names information at the network layer decoupling request resolution from data transfer in both time and space. The asynchronous nature of the Publish/Subscribe architecture simplifies resynchronization after MN handoffs and greatly facilitates mobility. However, clean-slate ICN architecture proposals such as PURSUIT have one significant drawback in that the network stack in every $\mathrm{MN}$ and server, together with application network interface code, have to be replaced. Therefore, IP-over-ICN [16] has emerged as a solution that aims at enabling individual operators to enhance their services by deploying a gatewaybased architecture; this offers improved IP-based services with an ICN infrastructure at its heart without incurring any changes to the end-user equipment that use existing IP protocol stacks and connectivity. The combination of the opportunities arising from the path-based forwarding, with its direct path possibilities, and the backward compatibility of the IP-over-ICN solution poses the question: can this new form of delivery architecture improve the performance of IP mobility?

In this paper, we answer the aforementioned question by virtue of proposing a novel network-based mobility management approach using an IP-over-ICN network where an efficient path-based forwarding solution is provided in the core of the network, while exposing backward compatible IP communication at the edges. Similar to PMIPv6, our solution targets intra-domain, mobile networks with infrastructure managed by a single operator. Although, we use terms common to cellular systems our solution is equally applicable to complementary large-scale structured wireless LAN (WLAN) systems [17] [18] with suitable adaptation. In the proposed solution, no traffic anchoring is required to support mobility at the network core, and no $\mathrm{MN}$ equipment modification or user interaction is required at the network edges. To evaluate our proposal, we analyze the mobility costs in IP-over-ICN and PMIPv6 networks by deriving the corresponding cost functions in terms of signaling, packet delivery and handover latency costs. The evaluation is performed using both random-walks on random geometric graphs and realistic mobility traces in a real operator network in a large city environment. The results illustrate a significant saving that gives a reduction by $45 \%$ or more in the total network traffic cost when using the proposed solution compared to the costs imposed by PMIPv6.

The rest of the paper is structured as follows. Section 2 provides an overview of the utilized IP-over-ICN network architecture and the proposed mobility management solution. Section 3 formally models the cost analysis used for evaluating our proposed solution. Section 4 presents and discusses the modelling and simulation results, while Section 5 provides a survey of related work. Finally, Section 6 draws our conclusions. 


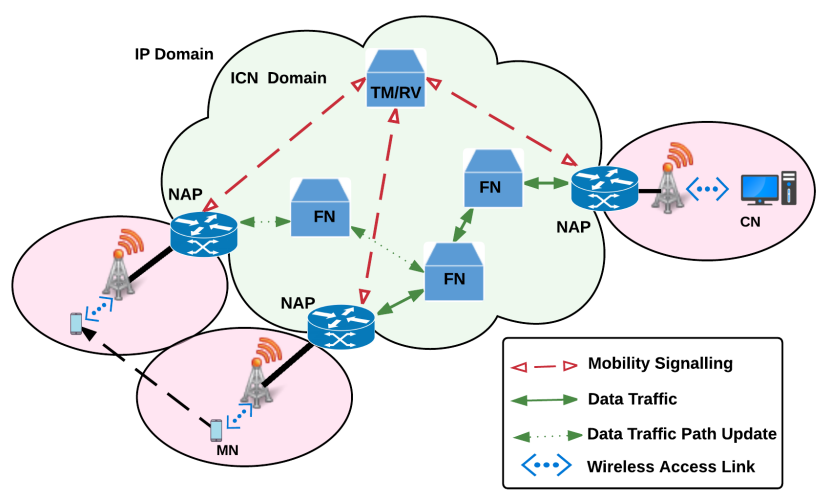

Fig. 2: IP-over-ICN Architecture and Mobility Management Overview.

\section{IP-OVER-ICN TO SUPPORT MOBILITY}

As emphasized in the previous section, this paper uses a path-based approach to achieve its benefits. However, this path-based approach needs some form of architecture to manage the interface between IP forwarding and the pathbased forwarding. An ICN architecture is utilized here as existing work has developed a suitable control-plane for the path-based forwarding. The proposed IP-over-ICN architecture follows a gateway-based approach, where the access from the user device to the network uses existing IP-based protocols, such as HTTP, CoAP, TCP or IPv4/v6, while the Network Attachment Point (NAP) serves as an entry point to the ICN network and maps the chosen protocol abstraction to ICN. The ICN core employs a Publish-Subscribe paradigm [19] for information dissemination that names information at the network layer, arranging individual information items into a context named scoping. In the context of an IP-over-ICN architecture the ICN names are simply the IP addresses of the end-systems or the fully qualified domain names (FQDNs) used by the end-systems. Scopes allow information items (here IP addresses or FQDNs) to be grouped according to application requirements, for example different categories of information. Relationships between information items and scopes are represented as a directed acyclic graph of which leaves represent pieces of information and inner nodes represent scopes. Each node in the graph is identified with its full path starting from a root scope, a more detailed explanation of it is given in [19].

There are three main functional entities that compose the ICN architecture as shown in Fig. 2: the Rendezvous (RV), the Topology Manager (TM) and Forwarding Nodes (FN). The RV is responsible for matching publications and subscriptions of information items while the TM is responsible for constructing a delivery tree for the information object. This delivery tree is encoded in a forwarding identifier (FID) which is sent to the publisher so that it can forward the packets containing the information object to the subscriber. Note that, the FID encodes a tree to allow for possible multicast delivery, where unicast is a trivial subset of a tree. In this paper, we will ignore the multicast capability as we wish to compare with existing mobile IP solutions that are usually focused on unicast. In the network, there are also Forwarding Nodes (FN) that simply forward the information object to the subscriber using the specific FID generated for this transmission [20]. Throughout this paper, the TM and RV functions are assumed to be residing in the same entity for the sake of simplicity, although they may be distributed or separated to support a scalable and resilient solution.

It should be noted that, as with IP networks, the pathbased forwarding needs to be able to cope with traffic engineering issues such as traffic management and resiliency. Both of these are out of the scope of this paper but research shows that these can be improved using this path-based approach compared to traditional IP networking [20], [21]. One feature of the path-based forwarding is that it enables stateless multicast switching using a mechanism such as LIPSIN [11] or BIER [13]. More recently it has been shown that LIPSIN can be realised using standard SDN switches without modification, allowing a truly stateless multicast switching solution that uses path-based forwarding and offthe-shelf networking components [12]. It should be pointed out that, although the path-based forwarding allows stateless switching, the per-tree state still needs to be maintained in the NAPs; unlike IP multicast, where the per-tree state has to be maintained in all routing elements.

The IP-over-ICN operation uses Publish/Subscribe (Pub/Sub) semantics for carrying IPv4/v6 datagrams over the ICN network. First, a naïve Pub/Sub signaling description will be given, to show the underlying principle, although in a likely deployment there will be optimizations to this naïve signaling that will be explained later. In the first instance, ICN signaling may sound complex. However, it must be remembered that this needs to be compared to the protocols required for an IP network application including DHCP, DNS, routing etc. to name but a few for general support, and of course the PMIPv6 signaling that is the specific protocol relevant to this paper. ICN signaling may be likened to this support signaling.

\subsection{A naïve IP-over-ICN signaling approach}

To explain the underlying IP-over-ICN principle, a naïve signaling approach is first presented as described in [16] for a system without mobility. The ICN uses Publish/Subscribe as its native communication mechanism; this does not immediately convert to the send/receive mechanisms used by IP datagrams that underpin TCP/IP communication. Consequently, this description describes how the IP is mapped to the ICN Publish/Subscribe model. ICN uses a namespace to facilitate communication, this namespace may be used to label any type of information. In an IP-over-ICN scenario, an IPv4/v6 address simply becomes an ICN name; the NAP uses Publish/Subscribe semantics to map IP datagrams to ICN names and then uses these names to forward IP datagrams as ICN information items through the ICN network. To aid the description, we will consider an IP client connected to what we describe as a client NAP (cNAP) and an IP server connected to a server NAP (sNAP). The cNAP and sNAP are only descriptive notations used for the naïve description, in practice a NAP will perform functions for any client or server connected to it so that, in practice, a NAP performs as both a cNAP and sNAP. The NAPs may form part of an access gateway or may be integrated into 


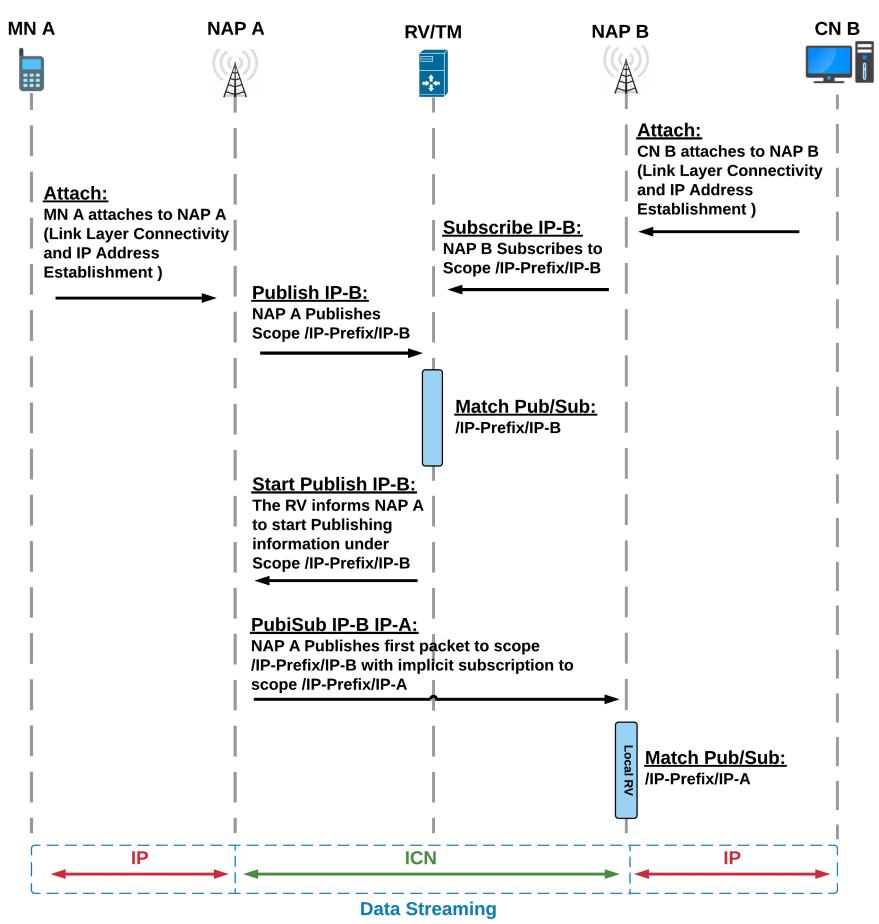

Fig. 3: Sequence Diagram of Session Establishment in IPover-ICN Networks.

IP routers that are gateways between the ICN network and the ICN network. In this paper, they are generally shown separately from the wireless Base Stations to simply show that they provide a different functionally.

An sNAP providing connectivity to an IP server is said to subscribe to receiving packets destined for the IP server, this subscription state is registered in the domain RV. Then if an IP client wishes to send data to the IP server the cNAP is said to publish the IP datagrams to the IP server NAP. To actually forward the IP datagrams, the cNAP requires an FID for the forwarding function which is obtained through Pub/Sub matching. Pub/Sub matching occurs in the RV when both a publisher and subscriber are registered for a unique ICN name, in this case the ICN name is the server IP address. Thus, when the cNAP registers the publication to the RV, the RV notes the match and requires the TM to send an appropriate FID to the cNAP so that it can publish (transmit) the data to the sNAP. In the naïve approach, when the IP server replies this whole mechanism can be reversed so that IP datagrams can flow in the reverse direction as well. When the client/server stop communicating (e.g. after a TCP FIN or after a suitable time-out) the Publish/Subscription matching state can be removed from the RV as communication is no longer required. The server subscription state is still maintained so that future IP clients can start a new communication.

In practice this naïve signaling approach is inefficient in terms of both state requirements in the RV and the number of signaling messages. Consequently, a practical system implements signaling optimizations including combining the cNAP publication message with an implicit subscription and only keeping the server subscription state in the RV. These optimizations are included in the signaling described in Section 2.3.

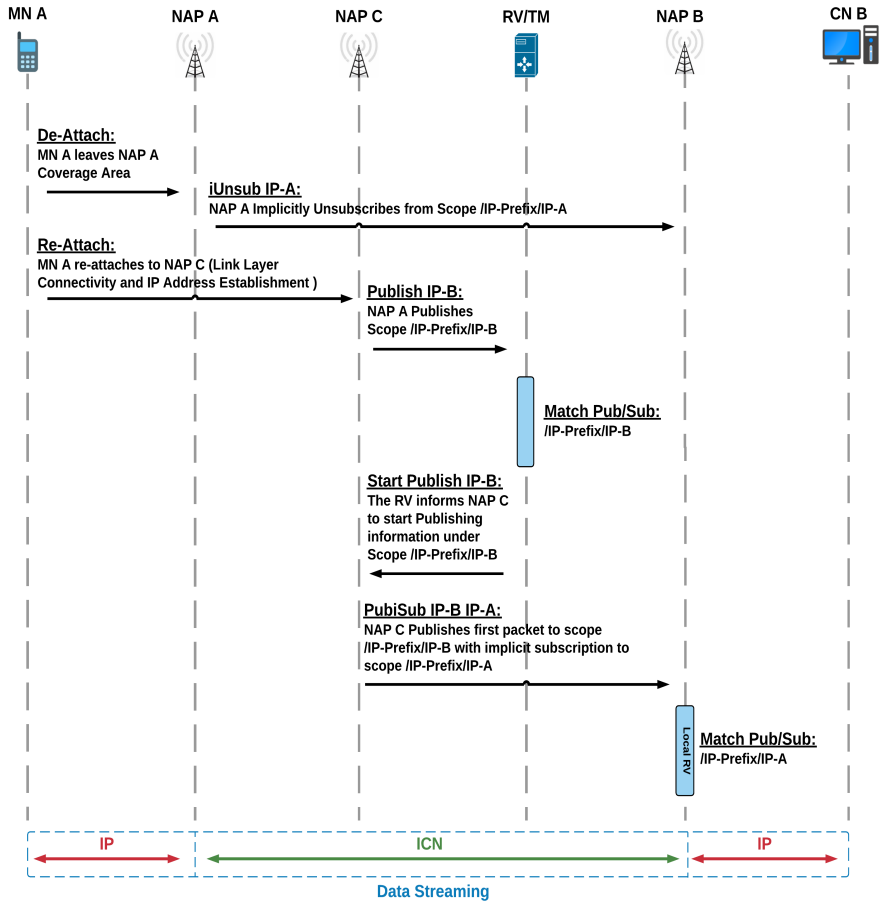

Fig. 4: Sequence Diagram of Handover Management in IPover-ICN Networks.

\subsection{IP-over-ICN Mobility Management}

For mobility management in IP-over-ICN, we propose that the NAP could serve as a MAG that performs the mobility management on behalf of a mobile node. The NAP occupies a key role in both MN network attachment and IP/ICN abstraction and interfacing. Therefore, it is a natural point for detecting the mobile node's movements to and from the access link since it resides at the access link where the mobile node is attached. On the ICN side, we propose that a centralized TM initially sets up the required routing state in the network and creates FIDs to forward packets from a NAP to every other NAP according to the deployed routing algorithm. All the NAPs receive their specified FIDs and populate a local table containing the complete set of FIDs required to reach any other NAP in the network. In IP-over-ICN, the mobile node will receive the IPv4/IPv6 address that the NAP locally assigns, and the NAP will act on behalf of the mobile node as the publisher or the subscriber towards the ICN. The ICN represents the network structure of IP addresses in a namespace under a unique root scope and an IP address of any device is interpreted as an appropriate ICN name under this scope. This means that the NAP will be ready to receive any information being sent to the assigned IP address by determining the appropriate ICN name according to the defined namespace. Therefore, any IP packet being sent to an IP address allocated to an IP device will arrive at the NAP serving it as an ICN-compliant notification to a subscription to this IP address (represented as an appropriate ICN name) [16]. The proposed IP namespace includes a network prefix scope identifier that serves as a root identifier and represents the IP network prefix allocated to serve the subject network domain. Under this root scope, there exists a so-called IP scope that represents the individual IP addresses allocated to IP endpoints that 
exist within the domain. These identifiers are formed by hashing a fully qualified IP address into a single 256 bit identifier.

For IP-over-ICN networks, end-node IP address sustainability can be maintained using any suitable IP autoconfiguration mechanism suitable for the network infrastructure deployed. One example is the Dynamic Host Configuration Protocol (DHCP) where every NAP can act as a DHCP server serving the entire subnet deployed in the IP-over-ICN domain. We propose that every DHCP server can be configured to only assign local addresses (for MNs that locally attach to the NAP) from a specific pool within the subnet, while it assigns addresses from outside the pool only to MNs that have previously been allocated an IP address at a previous NAP and intentionally ask for this specific IP address at the new NAP. This ensures that no IP address conflict would happen when the MN moves between NAPs. When a MN moves to a new NAP and goes into the DHCP RENEWING state, it would simply send a DHCPREQUEST message including the previously assigned IPv4 home address in the "Requested IP Address" option. The DHCPREQUEST is sent to the address specified in the Server Identifier option of the previously received DHCPOFFER and DHCPACK messages. The DHCP server would then send a DHCPACK to the MN to acknowledge the assignment of the committed IPv4 address following RFC2131 [22] and RFC5844 [7]. Each DHCP server on every NAP is configured to have the same IP address throughout the network, enabling the DHCPREQUEST message to be automatically sent to the available DHCP server on the access link without any delay. To facilitate IP address reuse, we propose that the RV keeps track of all the IP addresses used to maintain Pub/Sub relations in the network and sends periodic reports to all DHCP servers notifying them of abandoned IP addresses.

Note that in the discussion above, and in this paper in general, we consider the scenario where the IP address is owned by the operator and not shared across operators, i.e., a single operator, intra-domain scenario. Where this is not true, multiple operators using the same IP-overICN approach could signal between their RV's to organise handover between domains. Alternatively, if not using IPover-ICN, a mapping between the RV and the mechanism used in the external network is required.

On the link layer, a number of metrics exist to indicate the quality of connection and are used to indicate when mobility is occurring. One of these metrics is the Received Signal Strength Indicator (RSSI) which we use in this paper, alternatively, other predictors of mobility could be used, but their investigation is out of the scope of this paper. The RSSI value is part of the data transmitted by all mobile user equipment units. It is intended as a means to obtain a relative indication of the quality of connection that exists between the MN and the Base Station it is connected to in the cellular network. This could be used as the trigger for movement described in this example. Which NAP a client connects to is almost entirely determined by the MN itself. Thus, when a client is given a choice between multiple NAPs offering the same service, it will always choose the NAP with the highest RSSI. On the other hand just like the initial association sequence, when a $\mathrm{MN}$ is moving it also uses RSSI to determine when to disassociate from a NAP and associate with another.

\subsection{Signaling for anchorless mobility in IP-over-ICN}

In Section 2.1 a simple IP-over-ICN signalling mechanism was presented, however, that description did not support mobility and is inefficient. Here a full IP-over-ICN signaling mechanism is developed that supports mobility and optimizes the number of signaling messages. Fig. 3 shows a sequence diagram of the messages exchanged to establish a session between two IP endpoints in the proposed IP-overICN network. In this scenario, we assume that both the MN and the corresponding node $(\mathrm{CN})$ are in the same network domain. For simplicity, the examples assume a single subnet where a MN is likely to keep its IP address when moving among NAPs. The ICN core maintains session continuity by maintaining the same Pub/Sub matching relations at the RV even when a MN moves from one NAP to another. This forms one of the IP-over-ICN advantages compared to PMIPv6 networks for intra-domain scenarios because scalability is maintained by dividing and regionalizing the broadcast domain behind NAPs and routing is done through the ICN infrastructure using ICN semantics. This removes the scalability restrictions that would exist in an IP-core that would have to route / 32 host-routes for every host in the domain. In the IP-over-ICN case, the external IP network could be divided into subnets (maybe for address allocation reasons). The IP-over-ICN will treat the IP addresses in the same manner as a single subnet as forwarding within the ICN is orthogonal to the IP address allocation.

In the aforementioned scenario, we will first consider the signaling before mobility where MN A attaches to NAP A (Link Layer Connectivity and IP Address Establishment). NAP A extracts from the first packet sent from MN A towards $\mathrm{CN}$ B the source and destination IP addresses. NAP A translates the extracted addresses into appropriate ICN names according to the defined IP namespace before publishing the destination address Scope /IP-Prefix/IP-B to the domain $\mathrm{RV}$ on behalf of $\mathrm{MN} \mathrm{A}$. Upon receiving this publication, the RV then matches it with a previous subscription of NAP B to the same scope on behalf of $\mathrm{CN}$ $B$. The RV triggers NAP A to start publishing information to the identified subscriber located at NAP B. NAP A then looks up its local database for the appropriate FID to reach NAP B and uses it to send a PubiSub message directly to NAP B that includes the first data packet destined from $\mathrm{MN} A$ to $\mathrm{CN} B$ in addition to an implicit subscription to MN A's own scope /IP-Prefix/IP-A. NAP B utilizes its local $\mathrm{RV}$ to maintain a match Pub/Sub relation for scope /IPPrefix/IP-A, looks up its local database for the appropriate FID to reach NAP A and uses this FID to start publishing information to the identified subscriber located at NAP A. At this point $\mathrm{MN} A$ and $\mathrm{CN} B$ can commence data exchange. This procedure is only required for the first data packet exchange between the two IP endpoints. Subsequent data packets can be directly sent using the allocated FIDs.

Following the normal, static, IP-over-ICN scenario we now consider handover as part of mobility. Fig. 4 shows a sequence diagram of the messages exchanged to manage a handover procedure for MN A from NAP A to NAP C. After 
TABLE 1: Direction Probability Matrix and Steady State Probabilities.

\begin{tabular}{|c|c|c|c|c|c|c|c|c|c|c|}
\hline $\begin{array}{c}\text { Direction } \\
\text { Probability } p_{(k, j)}\end{array}$ & AP1 & AP2 & AP3 & AP4 & AP5 & AP6 & AP7 & AP8 & AP9 & $\begin{array}{c}\text { Steady-State } \\
\text { Probability }(\Pi)\end{array}$ \\
\hline AP1 & $1 / 4$ & $1 / 4$ & 0 & $1 / 4$ & $1 / 4$ & 0 & 0 & 0 & 0 & 0.100 \\
\hline AP2 & $1 / 4$ & $1 / 4$ & $1 / 4$ & 0 & 0 & $1 / 4$ & 0 & 0 & 0 & 0.100 \\
\hline AP3 & 0 & $1 / 3$ & $1 / 3$ & 0 & 0 & 0 & $1 / 3$ & 0 & 0 & 0.066 \\
\hline AP4 & $1 / 4$ & 0 & 0 & $1 / 4$ & $1 / 4$ & 0 & 0 & $1 / 4$ & 0 & 0.100 \\
\hline AP5 & $1 / 5$ & 0 & 0 & $1 / 5$ & $1 / 5$ & $1 / 5$ & 0 & $1 / 5$ & 0 & 0.133 \\
\hline AP6 & 0 & $1 / 6$ & 0 & 0 & $1 / 6$ & $1 / 6$ & $1 / 6$ & $1 / 6$ & $1 / 6$ & 0.166 \\
\hline AP7 & 0 & 0 & $1 / 4$ & 0 & 0 & $1 / 4$ & $1 / 4$ & 0 & $1 / 4$ & 0.100 \\
\hline AP8 & 0 & 0 & 0 & $1 / 5$ & $1 / 5$ & $1 / 5$ & 0 & $1 / 5$ & $1 / 5$ & 0.133 \\
\hline AP9 & 0 & 0 & 0 & 0 & 0 & $1 / 4$ & $1 / 4$ & $1 / 4$ & $1 / 4$ & 0.100 \\
\hline
\end{tabular}

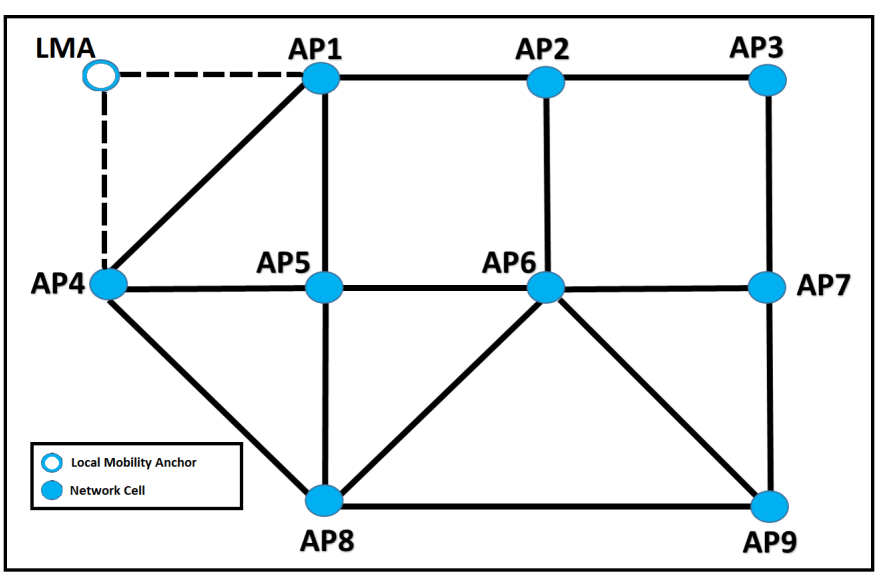

Fig. 5: Network Model Example.

initiating the handover procedure, the NAP on the previous link (NAP A) signals destination NAP B by sending an iUnsub message on behalf of MN A for it's own scope /IPPrefix/IP-A. This way the local RV at NAP B can remove the subscription state for MN A. According to this example scenario, MN A re-attaches to NAP C and re-establishes Link Layer Connectivity and IP Address allocation through DHCP which triggers NAP C upon receiving the first IP packet from MN A to Publish the destination Scope /IPPrefix/IP-B to the domain RV on behalf of MN A. The $\mathrm{RV}$ at this point re-matches the same publications and subscriptions established previously and triggers NAP C to start publishing information to the identified subscriber located at NAP B. NAP C then looks up its local database for the appropriate FID to reach NAP B and uses it to send a PubiSub message directly to NAP B that includes the first data packet destined from $\mathrm{MN} \mathrm{A}$ to $\mathrm{CN} B$ in addition to an implicit subscription to MN A's own scope /IP-Prefix/IP-A. NAP B utilizes its local RV to maintain a match Pub/Sub relation for scope /IP-Prefix/IP-A, looks up its local database for the appropriate FID to reach NAP C and uses it to start publishing information to the identified subscriber located at NAP C. At this point MN A and CN $\mathrm{B}$ can commence data exchange without further disruption using MN A's new location. Fig. 2 shows the participating entities and communication message flows for each of the control and data planes during mobility.

\section{Mobility Modelling and Cost Analysis}

In order to analyze the mobility behavior of mobile nodes in PMIPv6 and IP-over-ICN networks, a random walk mobility model is applied on connected graphs that represent the cellular network topology in terms of cells. This approach has been chosen due to the importance of the network topology and its influence on the total cost as described in the introduction. However, later, in Section 4.2 the architecture is also evaluated using actual mobility traces in a realistic cellular network to validate the analysis.

Fig. 5 shows an example network topology graph consisting of 10 nodes that is used to explain the details of the analysis performed. Given a random starting point, we select a random neighbor to move into (assuming equal transition probability to any neighbor for simplicity), then we select a neighbor of this new point at random, and move to it etc. The random sequence of points selected this way is a random walk on the graph. A random walk on a network graph of access cells possesses some unique distinctive properties that can be pointed out, including that of spatial homogeneity. This means that the transition probability between two points ( $x$ and $y$ ) on the graph should depend on their relative positions in space. This is obviously due to the fact that a mobile user at any given time can only move to a neighboring cell from any cell it is attached to. Also this implies that this random walk demonstrates the skip-free property, namely that to go from point $x$ to point $y$ it must pass through all intermediate points because it can only move one point at each step. In our analysis the cellular network is modeled as a connected graph whose nodes represent the coverage areas. This allows for flexibility in topology formation and cell shape assumptions from square and hexagonal cells to completely random topologies. Using a random walk on a connected graph to model user mobility leads to a discrete time finite Markov chain which provides a very practical and reliable way of estimating the location and direction probabilities of a moving user. The location probability represents the likelihood that a $\mathrm{MN}$ is located within the range of a specific cell at a given point in time, while the direction probability represents the likelihood that a $\mathrm{MN}$ is moving into the coverage area of a specific neighboring cell within the given set of neighboring cells at a given point in time. The Markov chain will be used to derive the global balance equations and also to introduce mobility rates into our mobility analysis. A random walk on a connected and undirected graph can be represented as follows [23]. $G=(V, E)$ is a connected, non-bipartite, undirected graph where $V$ are vertices that represent network cells and $E$ edges that represent the interconnections between the cells. Each cell, $k \in V$, has a set of neighbors $N_{k}=\{v: v \in V,(k, v) \in E\}$ with the number of neighbors 


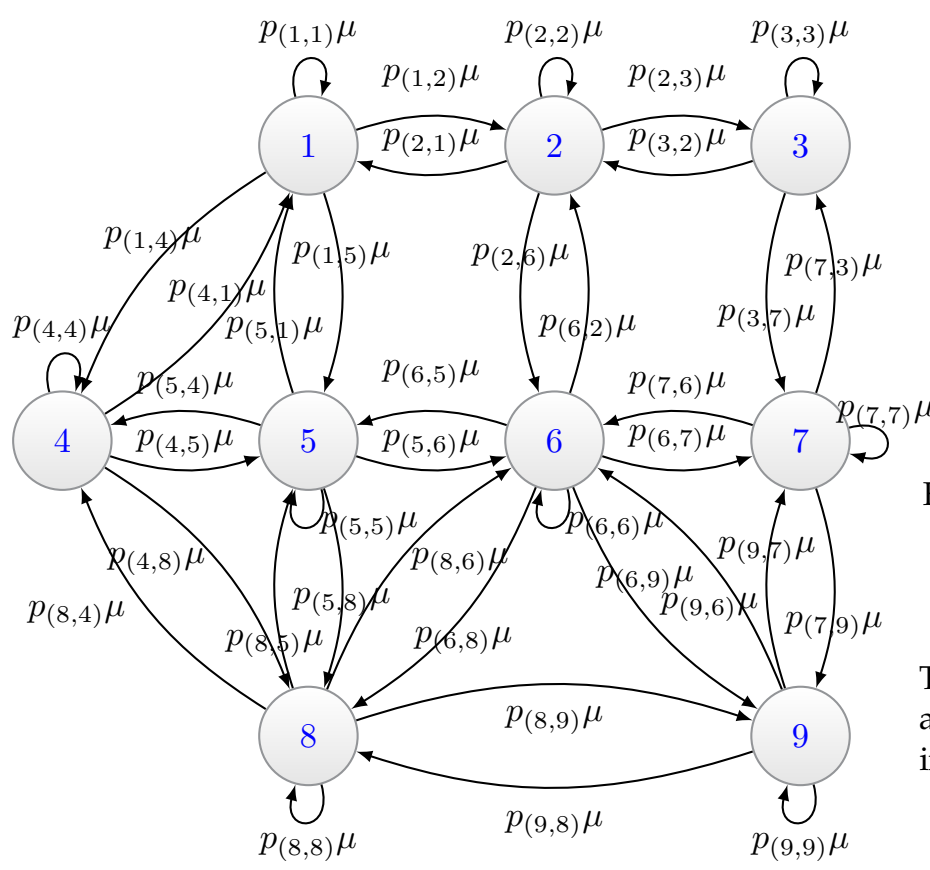

Fig. 6: Network Markov Chain Representation

denoted as $\left|N_{k}\right|$. This graph represents a Markov chain where the states are the nodes of $G$. Mobility is represented through elements $p_{(k, j)}$ of the direction probability matrix $\mathbf{P}=\left(p_{(k, j)}\right), \forall k, j \in V$, where $p_{(k, j)}$ is the probability that a $\mathrm{MN}$ was in the previous time slot within the range of a cell $k \in V$ and moves to a cell $j \in V$ in the current time slot. It is assumed that the neighbors are selected uniformly, at random. Thus, the probability of neighbor selection, $p_{(k, j)}$ depends on the degree of a node $k$ as follows:

$$
p_{(k, j)}= \begin{cases}1 /\left(\left|N_{k}\right|+1\right) & \text { if } j \in N_{k}, \\ 0 & \text { otherwise. }\end{cases}
$$

Given the direction probability from the equation above, there exists a unique steady-state location probability distribution vector $\Pi=\left(\pi_{1}, \pi_{2}, \ldots, \pi_{K}\right)$, such that $0 \leq \pi_{k} \leq 1$ for $1 \leq k \leq K$, where $K=|V|$ and $\pi_{k}$ represents the probability of a node being located at $k \in V$. The steady-state probability vector can be obtained by solving $\Pi=\Pi P$ and $\Pi e=1$, where $e$ is the $K \times 1$ matrix with all elements equal to 1 [24]. From our network model example in Fig. 5, the direction probability matrix and the steadystate probability vector are shown in Table 1.

The mobility on the connected graph above can be represented as a Markov process where states represent the traversed network cells and transitions between states representing flows of a Markovian process. Fig. 6 shows a complete Markov chain representation of the example network topology depicted in Fig. 5. The Markov process introduces the mean cell border crossing rate $\mu$ where in the analysis we assume that the mean cell border crossing rate is the same in all cells; this essentially assumes that mobility is homogeneous and that cells have the same area. Note that the local mobility anchor (LMA) has not been included in the Markov chain as it is not part of the mobility model as no MN transition into the LMA is permitted.

Assuming a system at steady state, the detailed balance equation for a user at state 1 (Network Cell 1) can be obtained as follows:

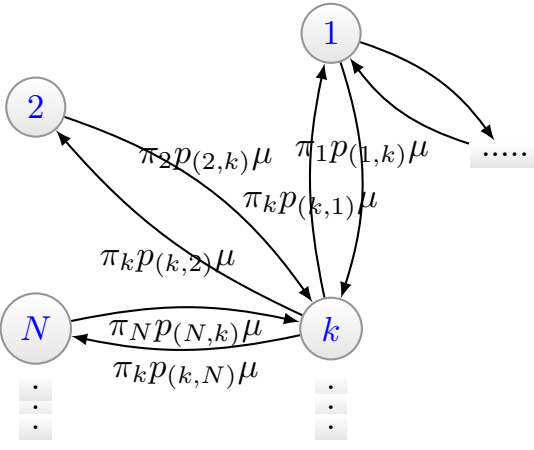

Fig. 7: General Markov Process for Markov Chain Mobility.

$$
3 \pi_{1} p_{(1, i)}=\sum_{j \in J} \pi_{j} p_{(j, 1)}, \quad \forall i \in J=\{2,4,5\}
$$

The general case for cell $k$, where the neighbors of the cell are $N_{k}$, is represented in Fig. 7. Thus, the specific example in (2) can be generalized as the global balance equation:

$$
\left|N_{k}\right| \pi_{k} p_{k} \mu=\sum_{j \in N_{k}} \pi_{j} p_{(j, k)} \mu \text { for } 1 \leq \mathrm{k} \leq \mathrm{K},
$$

where $p_{k}=1 /\left(\left|N_{k}\right|+1\right)$ is the generalized direction probability for a MN to move out of cell $k$.

TABLE 2: Summary of notation.

\begin{tabular}{|c||c|}
\hline Notation & Description \\
\hline$\mu$ & $\begin{array}{c}\text { Mobility Rate } \\
\text { into MAG } j \text { from } k\end{array}$ \\
\hline$p_{(k, j)}$ & $\begin{array}{c}\text { Direction Probabily that a mobile node is moving } \\
\text { into MAG k }\end{array}$ \\
\hline$\pi_{k}, \Omega^{\prime}$ & $\begin{array}{c}\text { Tocation Probability that a mobile node is attached } \\
\text { Network, respectively }\end{array}$ \\
\hline$\Upsilon, \Upsilon^{\prime}$ & $\begin{array}{c}\text { Mobility signaling Cost in a PMIPv6, } \\
\text { IP-over-ICN Network, respectively }\end{array}$ \\
\hline$\Lambda, \Lambda^{\prime}$ & $\begin{array}{c}\text { Mobility packet delivery cost in a PMIPv6, } \\
\text { IP-over-ICN Network, respectively. }\end{array}$ \\
\hline$h_{k, a}$ & $\begin{array}{c}\text { Number of hops between the MN initial MAG } k \\
\text { and the LMA }\end{array}$ \\
\hline$h_{j, a}$ & $\begin{array}{c}\text { Number of hops between the MN new } \\
\text { MAG } j \text { and the LMA }\end{array}$ \\
\hline$h_{s, a}$ & $\begin{array}{c}\text { Number of hops between the CN's MAG } \\
\text { and the LMA }\end{array}$ \\
\hline$R, R^{\prime}$ & $\begin{array}{c}\text { Average packet rate in a PMIPv6, } \\
\text { IP-over-ICN Network, respectively. }\end{array}$ \\
\hline$O_{k}, O_{k}^{\prime}$ & $\begin{array}{c}\text { Direct path packet overhead in a PMIPv6, } \\
\text { IP-over-ICN Network, respectively }\end{array}$ \\
\hline$h_{j, v}$ & $\begin{array}{c}\text { Number of hops between NAP } j \\
\text { and the RV /TM }\end{array}$ \\
\hline$h_{k, s}$ & $\begin{array}{c}\text { Number of hops between NAP } k \\
\text { and the destination NAP } s\end{array}$ \\
\hline$h_{j, s}$ & $\begin{array}{c}\text { Number of hops between NAP } j \\
\text { and the destination NAP } s\end{array}$ \\
\hline
\end{tabular}

\subsection{PMIPv6 Mobility Cost Analysis}

PMIPv6 is used as a reference model to compare the performance of the proposed IP-over-ICN mobility solution. PMIPv6 introduces two main entities that are involved in maintaining network enabled mobility support in a PMIPv6 domain which are the LMA that represents the networks central mobility anchor point and the MAG that acts as a mobility proxy on behalf of the mobile node. In order to update the LMA about the MN's current location, a Proxy Binding Update (PBU) message is sent from the MAG to the 


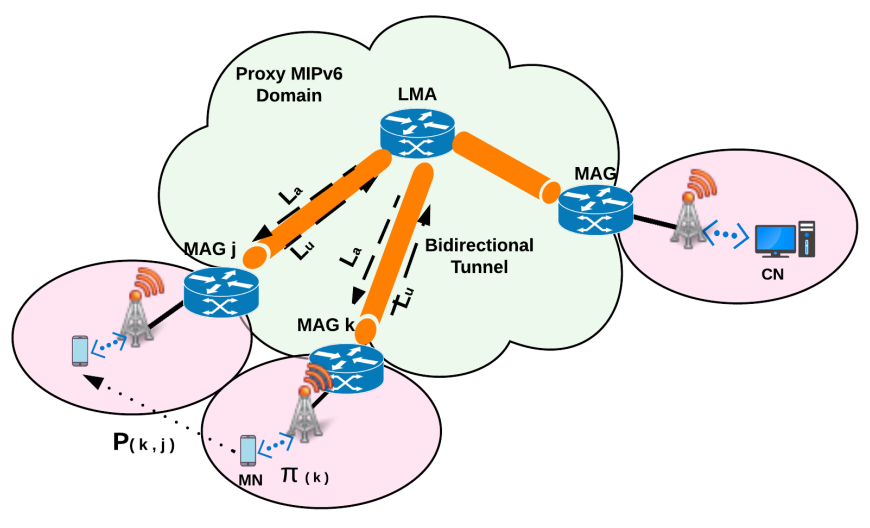

Fig. 8: Node Mobility in a PMIPv6 Domain.

MN's LMA. After accepting this PBU, the LMA sends back a Proxy Binding Acknowledgment (PBA) message to the MN's MAG that includes the MN's home network prefix. It also creates a binding cache entry into its binding cache table and establishes a bidirectional tunnel to the MAG. When the $\mathrm{MN}$ changes its point of attachment, the previous MAG on the previous access link detects the $\mathrm{MN}^{\prime}$ 's detachment from the link and signals the LMA to remove the existing binding and routing state for that MN. The new MAG, upon detecting the $\mathrm{MN}$ on its access link, signals the LMA to update the binding state. Therefore, for every MN transition from one MAG to another, two mobility signaling events are required, one for each of the two participating MAG's to the domain's LMA [25]. Fig. 8 shows a mobility scenario in a PMIPv6 domain with one $\mathrm{MN}$ and a static $\mathrm{CN}$. This scenario is considered in our mobility cost analysis which is general for either a static or mobile $\mathrm{CN}$.

It can be concluded from Section 3 that no general closed-form expression can be found for mobility cost analysis, due to the high dependability of the total cost on the network topological aspects. Therefore, the mobility cost analysis will be conducted by feeding the topological factors into the cost analysis equations. Specifically, the total cost for PMIPv6 $\Omega$ is split into signaling $\Upsilon$ and packet delivery cost $\Lambda$ as follows. ${ }^{1}$

$$
\Omega=\Upsilon+\Lambda
$$

where the signaling cost $\Upsilon$ is the signaling overhead for supporting mobility services for a MN. $\Lambda$ is the packet delivery cost that captures the tunneling and traffic anchoring overhead. $\Upsilon$ is calculated as the product of the size of mobility signaling messages and the hop distance. While $\Lambda$ is calculated as the product of the total packet size (including tunneling overhead) and the hop distance. The signaling cost $\Upsilon$ in Hops $\times$ Bytes/Sec can be calculated as:

$$
\begin{aligned}
\Upsilon=\sum_{k=1}^{K} \sum_{j \in N_{k}}\left\{\pi _ { k } p _ { ( k , j ) } \mu \left(h_{k, a}\left(\left|L_{u}\right|+\left|L_{a}\right|\right)\right.\right. \\
\left.\left.+h_{j, a}\left(\left|L_{u}\right|+\left|L_{a}\right|\right)\right)\right\}
\end{aligned}
$$

where $\pi_{k}$ is the location probability of a MN at MAG $k$; $p_{(k, j)}$ is the direction probability for the $\mathrm{MN}$ to move into MAG $j$ coverage area from MAG $k ; \mu$ the MN's mobility

1. Please refer to Table 2 for a list of the notations used in this paper.
TABLE 3: List of Mobility Messages and their Sizes.

\begin{tabular}{|c||c||c|}
\hline Notation & Description & Size in Bytes \\
\hline$L_{u}$ & Proxy binding update (PBU) & $76[1][26]$ \\
\hline$L_{a}$ & Proxy binding acknowledgement & $76[1][26]$ \\
\hline$\zeta$ & Average payload length & $1024[1][26]$ \\
\hline$\varphi$ & PMIPv6 tunnelling header & $40[1][26]$ \\
\hline$\ell_{u}$ & Implicit Unsubscribe (iUnsub) message & 166 \\
\hline$\ell_{r}$ & Publish Request message & 160 \\
\hline$\ell_{s}$ & Start Publish message & 166 \\
\hline$\ell_{p}$ & $\begin{array}{c}\text { Publish with Implicit Subscription } \\
\text { message (PubiSub) }\end{array}$ & 166 \\
\hline$\varphi^{\prime}$ & ICN payload packet header & 96 \\
\hline
\end{tabular}

rate of transition through a cell; $h_{k, a}$ is the number of hops between the LMA and MAG $k$; and, $h_{j, a}$ is the number of hops between the LMA and MAG $j$. As the MN changes its point of attachment, the previous MAG (i.e., MAG $k$ ) sends the de-registration PBU message to the LMA to inform the detachment of the MN at the access network managed by MAG $k$. As the new MAG (i.e., MAG $j$ ) detects the movement of the $\mathrm{MN}$, it registers the $\mathrm{MN}$ to the LMA by sending a PBU message. $\left|L_{u}\right|$ is the size of the proxy binding update (PBU) message sent from the MAG to the LMA and $\left|L_{a}\right|$ is the size of the proxy binding acknowledgment (PBAck) message. ${ }^{2}$ A list of mobility messages and their corresponding sizes is shown in Table 3 . If we set the proxy binding update and proxy binding acknowledgment size (in bytes) as

$$
\left|L_{T}\right|=\left|L_{u}\right|+\left|L_{a}\right|
$$

and substitute with $\left|L_{T}\right|$ in (5) we derive

$$
\Upsilon=\sum_{k=1}^{K} \sum_{j \in N_{k}} \pi_{k} p_{(k, j)} \mu\left|L_{T}\right|\left(h_{k, a}+h_{j, a}\right)
$$

The packet delivery cost $\Lambda$ is mainly used to investigate the tunneling and packet delivery overhead and is calculated as the product of total IPv6 packet size (including tunneling overhead) and the hop distance. The packet delivery cost for PMIPv6 $\Lambda$ in Hops $\times$ Bytes/Sec is given by

$$
\Lambda=\sum_{k=1}^{K} \pi_{k} R O_{k}
$$

where $\mathrm{R}$ is the average packet rate, and $O_{k}$ is the direct path packet cost in PMIPv6 which is obtained as

$$
O_{k}=h_{s, a}(\varphi+\zeta)+h_{k, a}(\varphi+\zeta)
$$

The parameter $h_{s, a}(\varphi+\zeta)$ is the direct path packet cost from the $\mathrm{CN}$ to the LMA and is equal to the number of hops between the CN and the LMA $h_{s, a}$ multiplied by the average data packet length in Bytes including tunnelling overhead $(\varphi+\zeta)$. On the other hand, $h_{k, a}(\varphi+\zeta)$ denotes the direct path packet cost from the MN (k) to the LMA and therefore the cost is equal to the number of hops between the $\mathrm{MN}$ and the LMA, $h_{k, a}$, multiplied by the average data packet length in bytes including tunneling overhead $(\varphi+\zeta)$. The complete path packet cost is the sum of the cost between the $\mathrm{CN}$ and the LMA and the MN, $k$ and the LMA.

2. In this paper, $|x|$ denotes the length of message $x$. 


\begin{tabular}{|c|c|c|c|c|c|}
\hline \multirow{2}{*}{ FID 32B } & \multicolumn{2}{|c|}{ Destinantion ICN ID 64B } & \multicolumn{2}{|c|}{ iUsub:Source ICN ID 64B } & Source \\
\cline { 2 - 6 } & Root Prefix Scope & IP Scope & Root Prefix Scope & IP Scope & EUI48 \\
\hline
\end{tabular}

(a) iUnsub Message $\left(\ell_{u}\right)$

\begin{tabular}{|c|c|c|c|}
\hline \multirow{2}{*}{ FID 32B } & \multirow{2}{*}{ Notification ID 64B } & \multicolumn{2}{|l|}{ Pub:Destination ICN ID 64B } \\
\cline { 3 - 4 } & & Root Prefix Scope & IP Scope \\
\hline
\end{tabular}

(b) Request Publish Message $\left(\ell_{r}\right)$

\begin{tabular}{|c|c|c|c|c|}
\hline \multirow{2}{*}{ FID 32B } & \multirow{2}{*}{ Notification ID 64B } & \multicolumn{2}{|l|}{ Pub:Destination ICN ID 64B } & Dest. \\
\cline { 3 - 5 } & & Root Prefix Scope & IP Scope & \\
\hline
\end{tabular}

(c) Start Publish Message $\left(\ell_{s}\right)$

\begin{tabular}{|c|c|c|c|c|c|c|}
\hline \multirow{2}{*}{ FID 32B } & \multicolumn{2}{|c|}{ Destinantion ICN ID 64B } & \multicolumn{2}{|c|}{ iSub:Source ICN ID 64B } & source & $\begin{array}{c}\text { Variable Size } \\
\text { Payload }\end{array}$ \\
\cline { 2 - 6 } & Root Prefix Scope & IP Scope & Root Prefix Scope & IP Scope & EUI48 & Payloan \\
\hline
\end{tabular}

(d) PubiSub Message $\left(\ell_{p}\right)$

\begin{tabular}{|c|c|c|c|}
\hline \multirow{2}{*}{ FID 32B } & \multicolumn{2}{|c|}{ Destinantion ICN ID 64B } & Variable Size \\
& Root Prefix Scope & IP Scope & Payload \\
\hline
\end{tabular}

(e) Data Payload Message $(\zeta)$

Fig. 9: IP-over-ICN Message Formats.

\subsection{IP-Over-ICN Mobility Cost Analysis}

The mobility messages in the proposed IP-over-ICN infrastructure are totally incurred within the ICN core. Fig. 4 shows the sequence of mobility messages that take place during handover in an IP-over-ICN domain. For simplicity we always assume in our analysis that only one end of the communication is mobile (MN) and that the $\mathrm{CN}$ is static and not generating any mobility signaling. This is realistic as most mobile users are accessing static services such as video content delivery and file downloading. However, our mechanism works equally well with both static and mobile $\mathrm{CNs}$. After initiating a handover procedure, the NAP on the previous link (i.e. NAP A) signals the destination NAP (i.e. NAP B) by sending an iUnsub message $\left(\ell_{u}\right)$ from the MN's own IP address scope. This enables NAP B to gracefully remove the subscription state for $\mathrm{MN}$ A from the $\mathrm{CN}^{\prime} \mathrm{s}$ IP address scope. This state was established prior to the handover at NAP B's local RV. Upon the MN re-attachment to a new NAP (NAP C), and after it establishes Layer 2 connectivity and IP address allocation, NAP $\mathrm{C}$ receives the first IP packet destined to the $\mathrm{CN}$ and sends a Publish request message $\left(\ell_{r}\right)$ to the domain $\mathrm{RV}$ requesting publication to the CN's IP address Scope. Upon receiving the publish request, the RV matches it with a previous subscription to the same address scope requested by NAP B and sends a start publish message $\left(\ell_{s}\right)$ to the NAP on the new link (NAP C). NAP C then locally looks up the appropriate FID to reach NAP B and uses it to send a PubiSub message $\left(\ell_{p}\right)$ to NAP $B$ that includes the first data packet from the $M N$ to the $\mathrm{CN}$ in addition to an implicit subscription to MN A's own IP address scope. The PubiSub message triggers NAP B to utilize its local Rendezvous to maintain a match pub/sub relation for the mentioned scope, looks up its local database for the appropriate FID to reach NAP C and uses it to start publishing information to the identified subscriber. At this point $\mathrm{MN} A$ and $\mathrm{CN} B$ can commence sending and receiving data payload messages $(\zeta)$. Fig. 9 illustrates the detailed message formats and sizes for the mobility messages needed in an IP-over-ICN setup.

The mobility signaling cost equals the size of the signaling messages multiplied by the number of hops. Therefore, the introduced signaling overhead is given by

$$
\begin{array}{r}
\Upsilon^{\prime}=\sum_{k=1}^{K} \sum_{j \in N_{k}}\left\{\pi _ { k } p _ { ( k , j ) } \mu \left(h_{k, s}\left|\ell_{u}\right|+h_{j, v}\left(\left|\ell_{r}\right|+\left|\ell_{s}\right|\right)\right.\right. \\
\left.\left.+h_{j, s}\left|\ell_{p}\right|\right)\right\}
\end{array}
$$

where $h_{k, s}$ is the number of hops between the previous NAP $k$ and the destination NAP $\mathrm{s}, h_{j, v}$ is the number of hops between the new NAP $j$ and the RV/TM and $h_{j, s}$ is the number of hops between NAP $j$ and the destination NAP $s .\left|\ell_{u}\right|$ denotes the size of an implicit unsubscribe (iUnsub) message sent from NAP $k$ to NAP $s$ when the MN initiates a handover. $\left|\ell_{r}\right|$ is the size of a publish request message sent from NAP $j$ to the RV/TM upon a change in the MN's NAP attachment requesting publication to the destination address scope. $\left|\ell_{s}\right|$ stands for the size of a start publish message sent from the domain RV / TM after a match pub/sub happens triggering NAP $j$ to start sending data packets to NAP $s$. Finally, $\left|\ell_{p}\right|$ is the size of a publish with implicit subscribe (PubiSub) message sent from NAP $j$ towards NAP $s$ including the first data payload in addition to an implicit subscription to the MN's address scope at the new location (NAP $j$ ). In the upcoming evaluations the payload size has been excluded from the $\ell_{p}$ message size as it is not considered a mobility signaling cost.

The packet delivery cost, $\Lambda^{\prime}$, is mainly used to investigate the packet delivery overhead and is calculated as the product of total packet size and the hop distance:

$$
\Lambda^{\prime}=\sum_{k=1}^{K} \pi_{k} R^{\prime} O_{k}^{\prime}
$$

where $R^{\prime}$ is the average packet rate in an IP-over-ICN network, and $O_{k}^{\prime}$ is the direct path packet overhead in IPover-ICN obtained as

$$
O_{k}^{\prime}=h_{s, k}\left(\varphi^{\prime}+\zeta\right)
$$

where $h_{s, k}$ is the number of hops between NAP $s$ where the $\mathrm{CN}$ is attached and NAP $k$ where the MN is attached, and $\varphi^{\prime}$ is the size of the ICN packet header. Finally $\zeta$ is the average payload length in Bytes.

\subsection{Handover Latency Analysis}

In this section, we analyze the latency differences between PMIPv6 and IP-over-ICN. To allow a simple analysis, latency is interpreted in terms of number of messages exchanged, processes required and hops traversed to facilitate a successful mobility handover. According to the sequence diagrams in Fig. 10, we assume that $p$ denotes message processing time, $m$ denotes the time to exchange a message and $h$ denotes the number of hops that a message traverses. For simplicity we will assume that $p$ and $m$ are represented in arbitrary time units with $p=m=1$ time unit, i.e., that a link transmission delay is comparable to forwarding delay. Therefore, for PMIPv6, the handover latency cost $T_{c}$ according to the message sequence in Fig. 10(a) and the hop notations in Table 2 would be

$$
T_{c}=5 p+m h_{k, a}+2 m h_{j, a}
$$




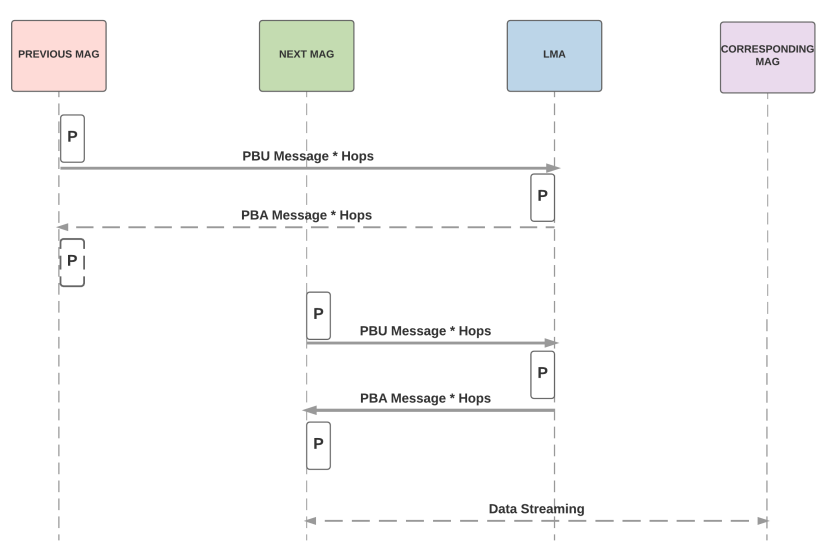

(a) PMIPv6.

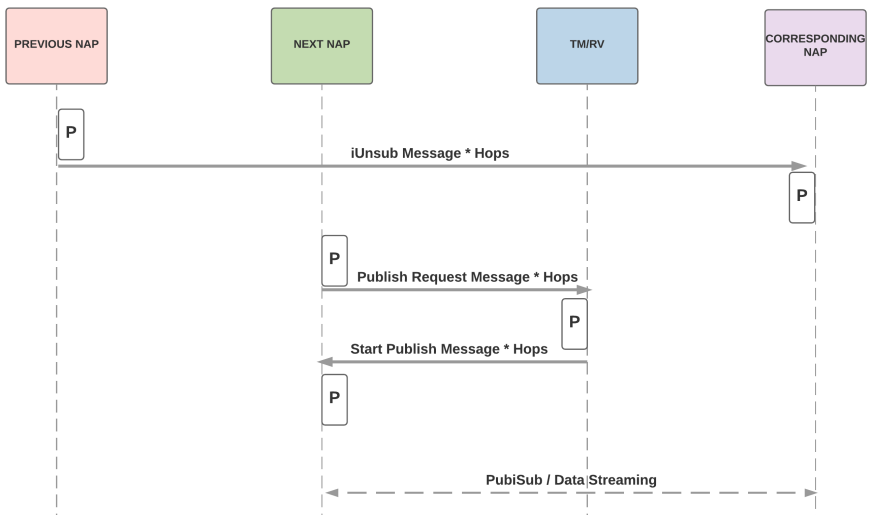

(b) IP over ICN.

Fig. 10: Sequence Showing Handover Latency.

The PBA message and its subsequent processing in dashed line (according to Fig. 10(a)) between the LMA and the previous MAG has not been added to the latency cost in (13) as it does not impact the time consumed by the MN to re-establish the session on the new MAG. For IP-over-ICN, the latency cost $T_{c}^{\prime}$ according to the message sequence in Fig. 10(b) and also referring to the hop definitions in Table 2 would be

$$
T_{c}^{\prime}=5 p+m h_{k, s}+2 m h_{j, v}
$$

where the PubiSub message sent at the end of an IP over ICN handover has not been added to the latency cost in (14) as it carries the MN's first data payload and therefore does not incur any extra latency. Although, (13) and (14) have the same number of node processes, the costs $T_{c}$ and $T_{c}^{\prime}$ are not necessarily equal to each other, as the path lengths may not be equal. If the LMA (in PMIPv6) and the RV/TM (in IP-over-ICN) are the same location, then the last term may be the same in both cases, however, the middle term differs as $h_{k, a}$ represents the source to anchor hop-count, whereas $h_{k, s}$ represents the source to $\mathrm{CN}$ hop-count.

\section{Mobility Management Simulation and Cost Evaluation}

To evaluate the performance of the proposed IP-overICN mobility solution and show the significance of the established analytical model; a packet level discrete time event simulation of both PMIPv6 and IP-over-ICN has been conducted in $\mathrm{R}$. The built simulation has been used to investigate the mobility costs (mainly mobility signaling, packet delivery and handover latency costs) with different scenarios and compare the results with those of our analytic model. The same central node was used to represent the LMA and TM/RV in all cases to ensure valid cost comparisons. In our traffic model, we assume that all the users in the network exchange video data with an arrival rate of 1 Mbps following a Poisson distribution.

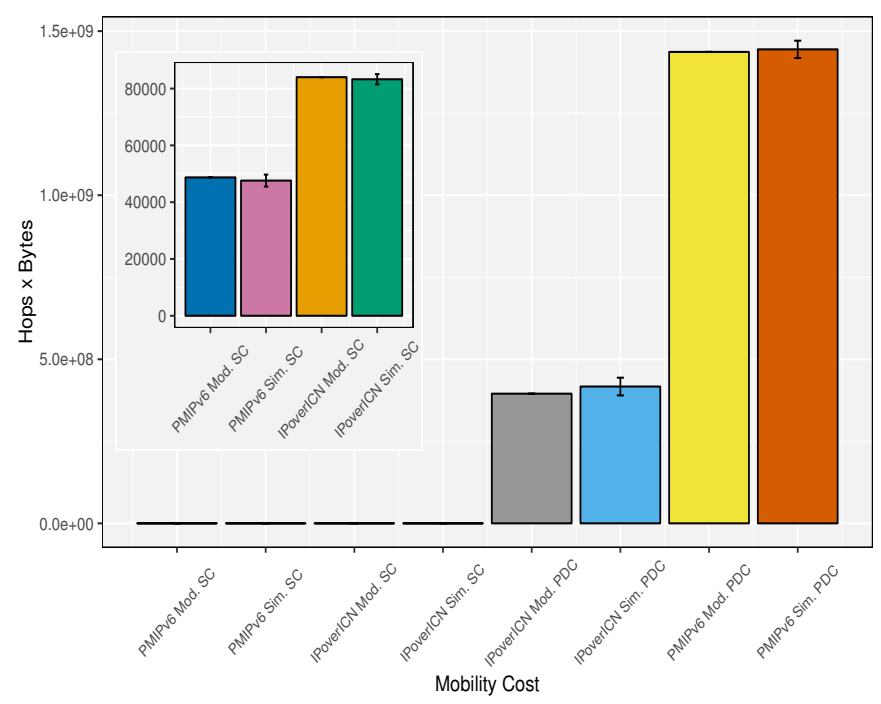

Fig. 11: Modelling (Mod.) and simulation (Sim.) of a single $\mathrm{MN}$ at $70 \mathrm{mph}$ with total packet delivery cost (PDC) and signaling cost (SC) using the example topology of Fig. 5. The error bars show the $95 \%$ confidence interval of the simulation results.

\subsection{Simulating Wireless Networks with Random Topologies and Mobility Behaviour}

In this simulation environment, random geometric networks have been used to represent network topologies to ensure spatial homogeneity of the positions of the MAGs and NAPs. Various network topology sizes with a different number of nodes (MAG's and NAPs) have been simulated with varying node degree. Both MAG's and NAPs have been simulated using a circular coverage area with a radius of $500 \mathrm{~m}$. A random walk mobility model has been used to capture user mobility with various speed values ranging from pedestrians moving at a rate of $3 \mathrm{mph}$ to vehicles travelling at $70 \mathrm{mph}$. Initial user locations are randomly distributed using a uniform random distribution. Every simulation experiment was run for 1800 seconds and repeated 20 times with results collated after reaching a steady state. 


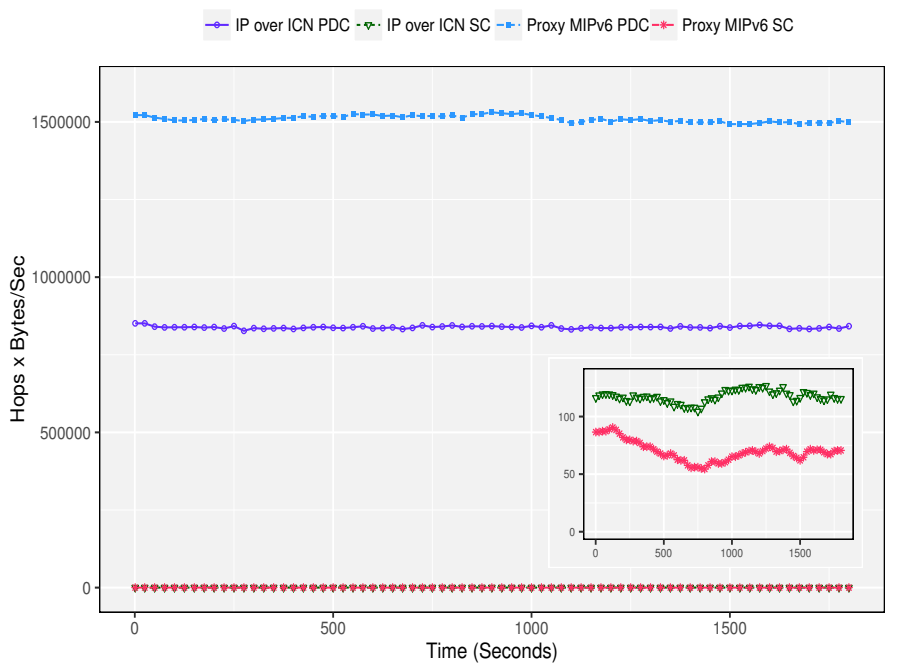

(a) Single Speed $(70 \mathrm{mph})$.

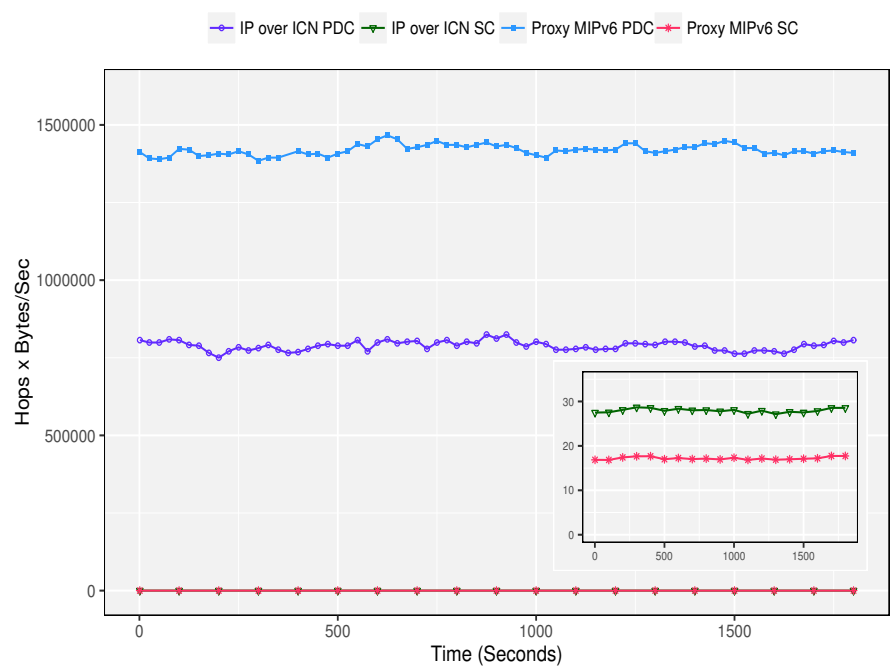

(b) Random Speed (3 - $70 \mathrm{mph}$ ).

Fig. 12: Simulation of $50 \mathrm{MNs}$ with average packet delivery cost (PDC) and signaling cost (SC) using a Geometric Random Topology of 100 nodes.

\subsubsection{Validating the Analytical Model Through Simulation}

The first simulation results use the topology example in Fig. 5 to compare the performance with those obtained from the cost analysis functions and mobility model in the previous section. Our modeling results were calculated as follows. Assuming a vehicle moving at a constant velocity of $70 \mathrm{mph}$ through a circular coverage area with a radius of $500 \mathrm{~m}$, this would result in the vehicle spending 25.12 second in every cell it traverses at a mobility rate $(\mu)$ of $0.0391 / \mathrm{s}$. Therefore, applying the derived cost functions for PMIPv6 and IP-over-ICN in equations (7), (8), (10) and (11) to the network model example in Fig. 5; and utilizing the Markov mobility model in Fig. 6 while substituting the variables with the values in Table 3; yields in the following results. $\Upsilon=21.624$ Hops $\times$ Bytes $/$ Sec,$\Lambda=809176$ Hops $\times$ Bytes $/$ Sec, $\Upsilon^{\prime}=43.758$ Hops $\times$ Bytes $/$ Sec and $\Lambda^{\prime}=218400$ Hops $\times$ Bytes $/$ Sec. To compare the results with those that would be obtained from a simulation that uses the random walk mobility model, a single $\mathrm{MN}$ was simulated to move randomly with speed of $70 \mathrm{mph}$. Both the MN initial location and traversed paths were selected randomly from a uniform distribution. Fig. 11 shows the accumulative simulation and modeling results for the example topology in Fig. 5 over 1800 seconds. The results show that the modeling results fall within the $95 \%$ confidence intervals of the simulation results. This shows that although the Markovian model is an idealised representation of mobility, it performs very accurately and is an appropriate tool to model the mobility costs. Fig. 11 also shows both the total packet delivery and signaling costs for PMIPv6 and IP-over-ICN, and it is clear from the results that PMIPv6 imposes a higher packet delivery cost of more than three times that of IP-over-ICN reaching about $15 \times 10^{8}$ Hops $\times$ Bytes due to the longer traffic paths imposed by using a central LMA. Also from Fig. 11 it can be seen that IP-over-ICN imposes higher signaling cost than PMIPv6 reaching approximately $9 \times 10^{4}$ Hops $\times$ Bytes compared to about $4 \times 10^{4}$ Hops $\times$ Bytes incurred by PMIPv6.
But despite the signaling cost results, the high difference in magnitude of packet delivery cost between PMIPv6 and IP-over-ICN indicates that IP-over-ICN highly outperforms PMIPv6 in the total cost.

\subsubsection{Mobile Node Speed Variation}

The second set of results use random geometric networks of 100 nodes with average connection degree of 4 neighbors (between 1 and 8 neighbors for every NAP/MAG in the network). $50 \mathrm{MNs}$ were simulated to move freely and randomly within the network domain. Various node speeds have been used in this experiment ranging between pedestrian speeds of $3 \mathrm{mph}$ and highway speeds of $70 \mathrm{mph}$. The MN initial locations, traversed paths and speeds, were all selected randomly from uniform distributions. Fig. 12(a) shows the average packet delivery and signaling costs at $70 \mathrm{mph}$ for both PMIPv6 and IP-over-ICN. According to this comparison, PMIPv6 shows approximately double the packet delivery cost imposed by IP-over-ICN due to the central traffic anchoring. Although IP-over-ICN shows higher signaling costs, the high difference in figures between packet delivery cost and signaling cost implies that IP-over-ICN requires half the total cost of PMIPv6 in order to provide network enabled mobility support. Another simulation run is shown in Fig. 12(b) using random MN speeds ranging from 3 to $70 \mathrm{mph}$. The figure clearly shows that the same difference in performance is observed between IP-over-ICN and PMIPv6 in terms of packet delivery and signaling costs respectively although random MN speeds have been simulated. Figs. 13(a) and 13(b) show the total incurred mobility signaling and packet delivery costs respectively when different MN speeds are simulated individually. From the results it can be seen that mobility signaling cost has a positive relation with MN speed ranging from only 3720 and 5593 Hops $\times$ Bytes for PMIPv6 and IP-over-ICN respectively with MN speed of 3 


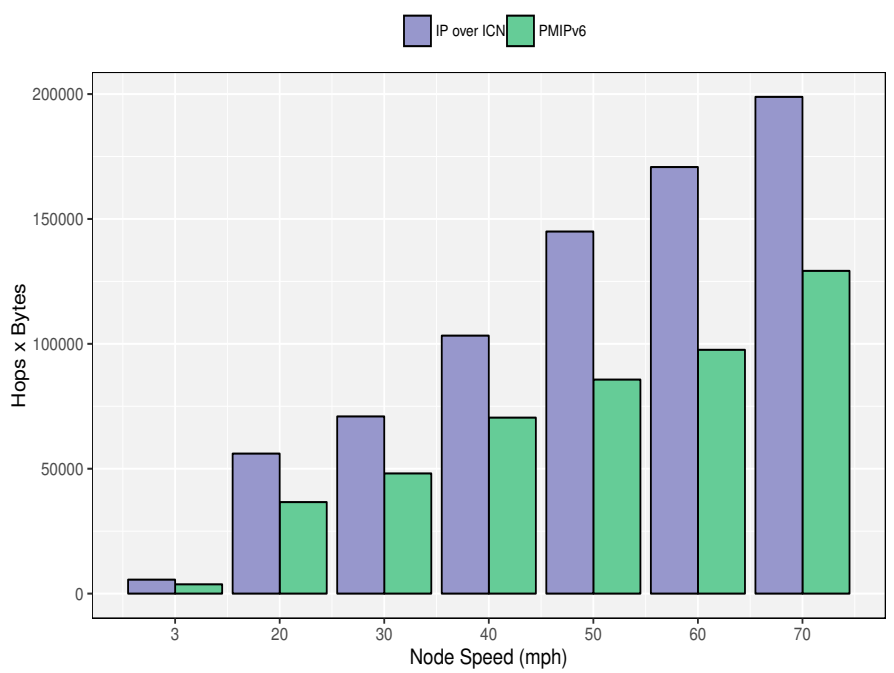

(a) Mobility Signaling Cost with Different Nodes Speed.

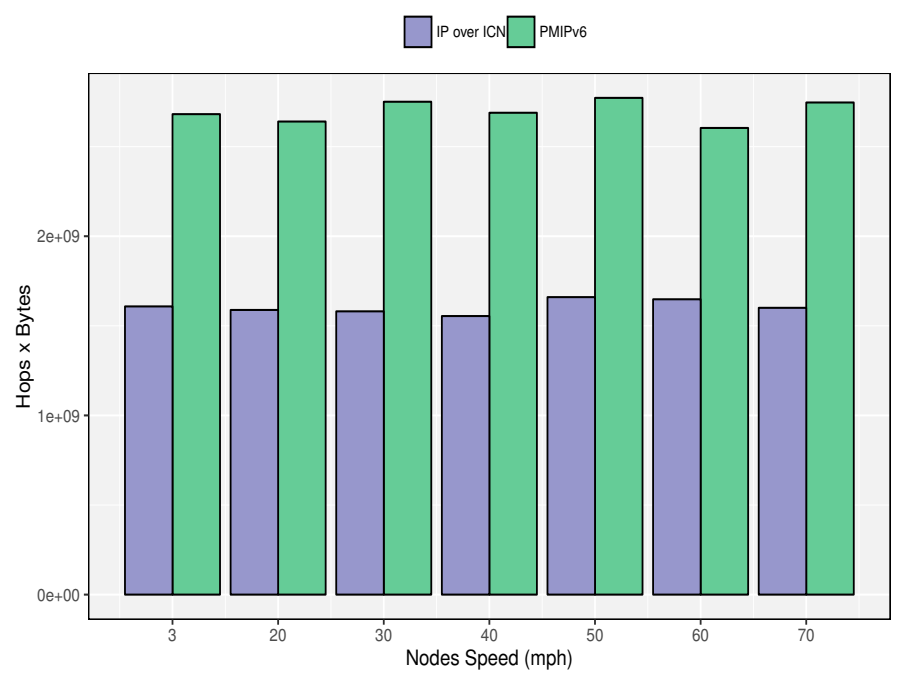

(b) Mobility Packet Delivery Cost with Different Nodes Speed.

Fig. 13: Simulation of $50 \mathrm{MNs}$ with total packet delivery cost (PDC) and signaling cost (SC) using a Geometric Random Topology of 100 nodes.

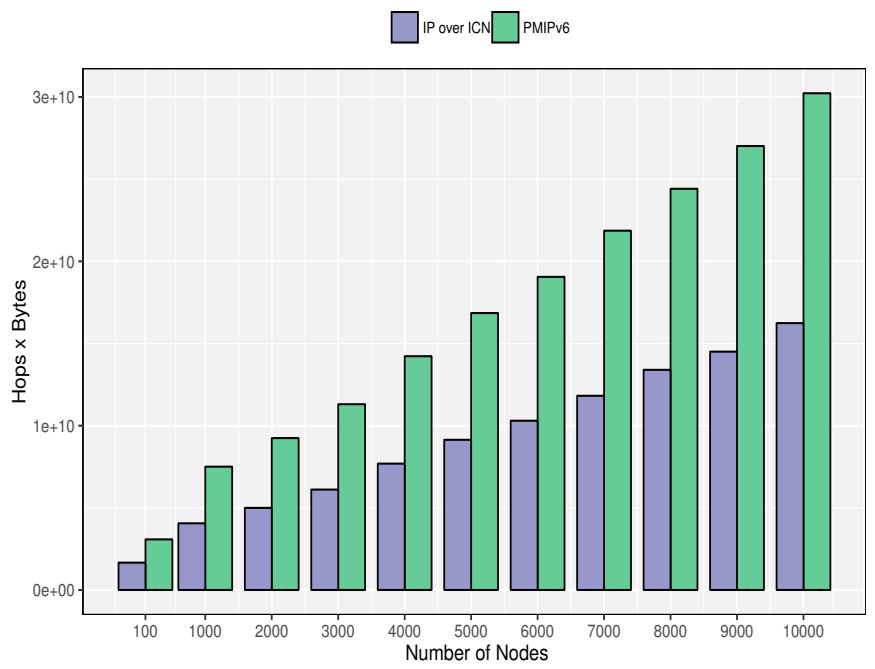

Fig. 14: Total Cost with Different Network Topology Sizes.

$m p h$ to about $1.3 \times 10^{5}$ and $2 \times 10^{5}$ Hops $\times$ Bytes for PMIPv6 and IP-over-ICN respectively with MN speed of $70 \mathrm{mph}$. On the other hand, the packet delivery cost is not influenced by any speed changes as seen in Fig. 13(b).

\subsubsection{Network Topology Size Variation}

The third simulation results are conducted using different network sizes to show how network size can effect the total cost. Random geometric networks ranging from 100 up to 10000 nodes have been simulated with average connection degree of 4 neighbors for every NAP/MAG in the network. $50 \mathrm{MNs}$ were simulated to move freely and randomly within the network domain with speed of $70 \mathrm{mph}$. Fig. 14 shows the total cost for both PMIPv6 and IP-over-ICN for each of the simulated topology sizes. It can be seen from the trends that IP-over-ICN always outperforms PMIPv6 in terms of the total cost required to support network-based mobility management with an improvement factor of at least 1.8

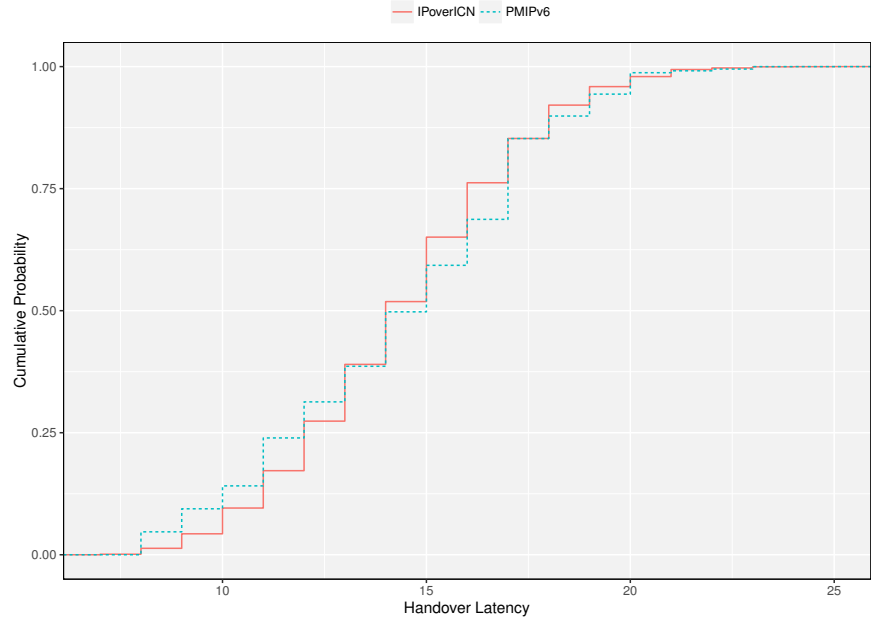

Fig. 15: Empirical Distribution Function of Handover Latency Costs in a Wireless Network.

due to the sub-optimal triangular routing mechanism of PMIPv6.

\subsubsection{Handover Latency Costs}

The fourth simulation experiment examines the handover latency in both IP-over-ICN and PMIPv6 networks using the same network topology of Section 6.2 with $100 \mathrm{MNs}$ moving freely and randomly within the network domain at $70 \mathrm{mph}$. Fig. 15 shows an empirical cumulative distribution function of the handover latency in both investigated domains. From this figure it can be seen that IP-over-ICN and PMIPv6 schemes have highly convergent distributions that are nearly identical at the upper range of handover latencies between 20-25 units time. This is due to the high similarity in the number of signalling messages and processes required to facilitate handover in both domains with minor differences in the traversed paths as outlined in section 5.3. This clearly illustrates that IP-over-ICN offers no extra cost in handover latency while our earlier results show significant savings on the data plane traffic. 


\subsection{Performance with Real Cellular Topology and Mo- bility Behaviour}

In this simulation environment, we use a publicly available realistic mobility dataset that describes vehicular mobility within the Cologne metropolitan region in Germany, in addition to the actual deployment of cellular infrastructure for the same region [27]. In order to approximate the coverage of individual Base Stations in the region, we run a Voronoi tessellation on the Base Station locations. The mobility dataset has been generated by coupling well known state of the art tools, in order to address all of the aspects required for a correct characterization of vehicular traffic. Realistic maps of the road topology in the region are extracted from the OpenStreetMap (OSM) database [28]. The microscopic mobility of vehicles is simulated with the Simulation of Urban Mobility (SUMO) software [29]. The traffic demand information on the macroscopic traffic flows across the Cologne urban area is derived through the Travel and Activity PAtterns Simulation (TAPAS) methodology [30]. This technique generates a realistic traffic demand, represented by a so-called Origin/Destination (O/D) matrix. In order to generate such an O/D matrix, real-world data collected in the Cologne region by the German Federal Statistical Office including 30,700 daily activity reports from more than 7000 households is processed [31]. Finally, Gawron's traffic assignment model [32] is run on the O/D matrix, so as to identify the actual route followed by each driver. The resulting mobility dataset mimics the daily movements of inhabitants of the Cologne metropolitan area for a period of 24 hours, comprising a total of more than 700.000 individual trips. The simulation run time was 1800 seconds during the morning peak hours in Cologne between 9:00 am and 9:30 am.

\subsubsection{Mobility Signaling and Packet Delivery Costs}

Fig. 16 shows the sum of packet delivery cost and signaling cost (average and total) for both PMIPv6 and IP-over-ICN. According to this figure, PMIPv6 shows approximately 1.8 of the total costs imposed by IP-over-ICN due to the central traffic anchoring. The results using real mobility traces and realistic network topology support the analysis and simulation presented in Section 4.1. And although IP-over-ICN imposes higher signaling costs that are approximately 1.7 the total signaling costs of PMIPv6, this barely affects the overall performance of our scheme as shown by Fig. 16 due to the high difference in value between packet delivery and signaling costs. Fig. 16 also compares the simulation total costs above with those obtained from applying the cost analysis and mobility model in Section 3 to the same realistic mobility traces and cellular topology used in the simulation. Our modeling results were calculated as follows. The direction probabilities from each cell to every other cell in the topology were populated using the realistic vehicular mobility patterns in the traces over the same period of time used in the simulation (1800 seconds). Then, the location (steady state) probabilities were calculated accordingly as explained in Section 3. The mobility rates were calculated from the realistic mobility traces by extracting the amount of time a user spends in every cell before attaching to another. Cell coverage areas were determined by running

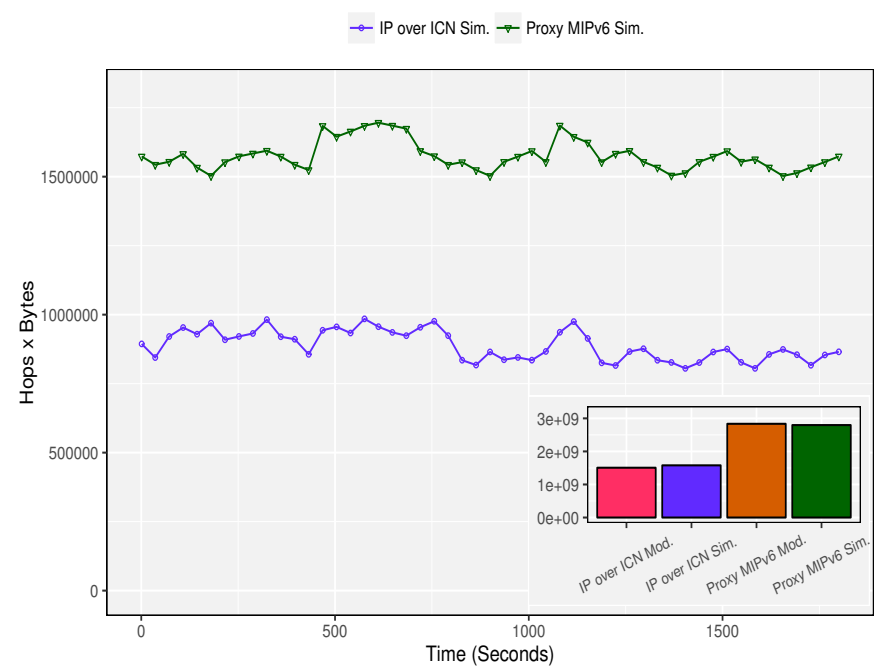

Fig. 16: Sum of Packet Delivery Cost and Signaling Cost (Average and Total) in a Cellular Network Obtained Through Simulation (Sim.) and Modelling (Mod.)

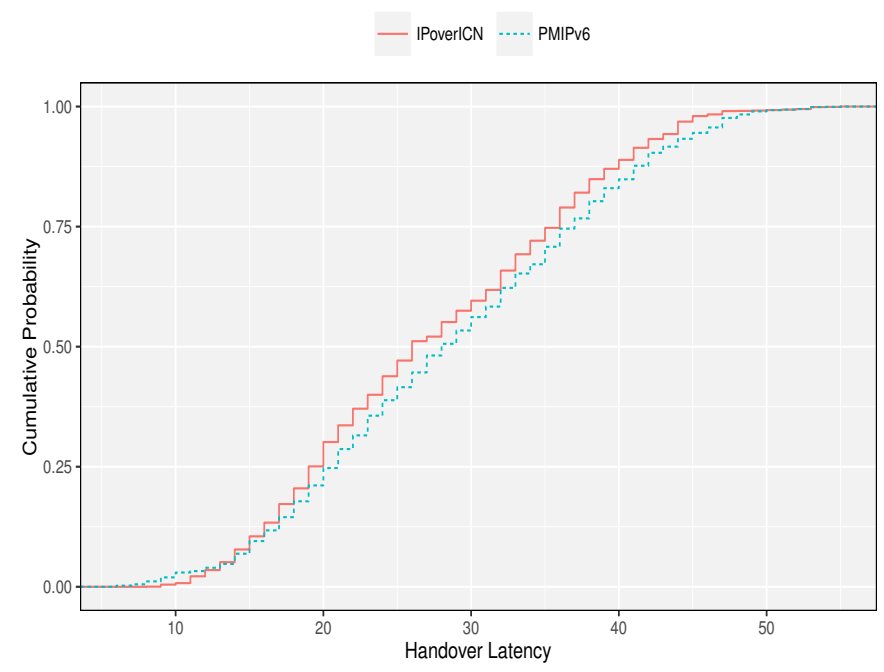

Fig. 17: Empirical Distribution Function of Handover Latency Costs in a Cellular Network

a Voronoi tessellation on the Base Station locations and the cost functions in Sections 3.1 and 3.2 were used to calculate the total costs. From Fig. 16, it can be observed that evaluation and modeling results are very close to each other.

\subsubsection{Handover Latency Costs}

This simulation experiment examines the handover latency in both IP-over-ICN and PMIPv6 schemes. Fig. 17 shows an empirical cumulative distribution function of the handover latency in both investigated domains. From this figure it can be seen that both IP-over-ICN and PMIPv6 have highly convergent distributions that are nearly identical. This is due to the high similarity in the number of signaling messages and processes required to facilitate handover in both domains with minor differences in the traversed paths as outlined in Section 3.3. Fig. 17 clearly illustrates that IP-over-ICN offers a slight gain in handover latency cost while earlier results show significant savings on the data plane traffic. 


\section{Related WORK}

PMIPv6 [4] has been adopted by the IETF to support network-based mobility in wireless networks by utilizing the LMA as a centralized mobility management entity on both the data and control plane. The LMA is responsible for maintaining reachability to the MN's IP address during the latter's movement between MAGs in the PMIPv6 domain. This is realised by updating the binding cache in a binding table and maintaining a tunnel to the MAG for packet delivery. On the other hand, 3GPP specifies the General packet radio service (GPRS) Tunneling Protocol (GTP) [33] to support mobility in cellular networks by anchoring user data plane traffic at the serving gateway (S-GW) and control plane traffic at the Mobility Management Entity (MME). GTP is an important IP/UDP based protocol used to encapsulate user data when passing through core network using GTP-U and also carries bearer specific signaling traffic between various core network entities using GTP-C. Also in efforts to significantly improve handover between heterogeneous network technologies, IEEE standards association has developed 802.21 [34] that defines a media-independent handover (MIH) framework. The standard defines the tools required to exchange information, events, and commands to facilitate handover initiation and handover preparation. A large number of efforts have focused on amendments, improvements and cost evaluation of the standards mentioned previously, we summarize the most significant of them below.

Distributed Mobility Management (DMM) efforts [35] [36] try to solve PMIPv6 drawbacks by evolving towards a flatter architecture using distributed anchoring, thereby providing a more efficient way to handle mobile traffic. In these approaches, although the LMA functionality is distributed into the network edges, they still perform traffic tunnelling and anchoring in a localized manner which does not eliminate the traffic overhead imposed to support mobility.

IEEE 802.21 Media Independent Handover (MIH) functionality assisted PMIPv6 solutions such as [37], aim at reducing handover latency and signaling cost in heterogeneous wireless networks. The Base Station with $\mathrm{MIH}$ functionality performs handover on behalf of the MN. The analytical evaluation shows that the proposed mechanism can outperform the existing mechanism in terms of handover latency and total number of over the air signaling messages. But despite that, the sub-optimal core routing problem remains unsolved.

Path-based forwarding architectures such as Software Defined Networks (SDN), bring new possibilities to improve the mobility management with lower traffic cost, better scalability and faster handover. Today, the most known approach is testing mobile flow entries against matching rule fields and finding a correct output action through every OpenFlow switch along the path, which has high costs in mobile flow management. Most of the proposed SDN architectures in wireless networks cannot be directly applied to large-scale networks due to this reason [38]. OpenFlowenabled proxy mobile IPv6 (OF-PMIPv6) is proposed in [39] where the control path is separated from the data path by performing the mobility control at the controller, whereas the data path remains direct between the MAG and the LMA in an IP tunnel form. This method achieves improved handover latency over conventional PMIPv6, while the data plane anchor problem is persistent. Other SDN efforts such as [40] propose rule caching mechanisms to tackle the limited rule space problem in existing SDN devices. Such approaches propose to support completely flat mobility architectures, but as a drawback, they incur additional processing complexity to manage the proposed caching mechanisms.

\section{CONCLUSION}

Efficient mobility management solutions are essential to accommodate the immense growth of mobile networks, users and generated traffic. In this paper, we introduced a novel, anchor-free, mobility management solution that utilizes a revolutionary path-based forwarding substrate to enable direct communication between the source and destination. We evaluated the cost of our solution through analytical modeling and simulations; and, compared it with the conventional PMIPv6. Evaluation results have shown that the delivery cost of our solution is approximately half of that incurred by the PMIPv6 counterpart; for similar or (in some cases) reduced end-to-end latency. Consequently, we have shown that significant resource saving can be achieved using our proposed solution.

By introducing the anchor point, PMIPv6 clearly violates network end-to-end transparency, and also introduces a network state (not flow-based, but device based), which is considered a drawback for processing, security as well as failure perspectives. Strictly speaking, IP-over-ICN still violates the transparency but at a much better point of the system, namely at the attachment points of both communication parties. This paper demonstrates that this is an improved point for the violation, as it allows optimal delivery paths, i.e., the same path that would be used if mobility had not occurred. This paper has used an IP-over-ICN solution as an embodiment to facilitate the proposed anchor-free mobility solution. However, the proposed mobility solution can be facilitated by any forwarding architecture that purely relies on path information stored in the forwarded packet for the end-to-end delivery; in this case, mobility simply results in partial re-computation of the path, with the opportunity to deliver the data over an optimal path after every handover operation.

\section{REFERENCES}

[1] J.-H. Lee, T. Ernst, and T.-M. Chung, "Cost Analysis of IP Mobility Management Protocols for Consumer Mobile Devices," IEEE Trans. on Consumer Electronics, vol. 56, no. 2, pp. 1010-1017, May, 2010.

[2] C. J. Bernardos, M. Gramaglia, L. M. Contreras, M. Calderon, and I. Soto, "Network-Based Localized IP Mobility Management: Proxy Mobile IPv6 and Current Trends in Standardization," Journal of Wireless Mobile Networks, Ubiquitous Computing, and Dependable Applications (JoWUA), vol. 1, no. 2/3, pp. 16-35, Sep., 2010.

[3] I. Soto, C. J. Bernardos, M. Calderón, and T. Melia, "PMIPv6: A Network-Based Localized Mobility Management Solution," The Internet Protocol Journal, vol. 13, no. 3, pp. 2-15, Sep., 2010.

[4] S. Gundavelli, K. Leung, V. Devarapalli, and K. Chowdhury, "B. Patil," Proxy Mobile IPv6," RFC 5213, Tech. Rep., 2008. 
[5] J.-H. Lee, J.-M. Bonnin, I. You, and T.-M. Chung, "Comparative Handover Performance Analysis of IPv6 Mobility Management Protocols," IEEE Trans. on Industrial Electronics, vol. 60, no. 3, pp. 1077-1088, March, 2013.

[6] F. Giust, C. J. Bernardos, and A. De La Oliva, "Analytic Evaluation and Experimental Validation of a Network-Based IPv6 Distributed Mobility Management Solution," IEEE Trans. on Mobile Computing, vol. 13, no. 11, pp. 2484-2497, Nov., 2014.

[7] R. Wakikawa and S. Gundavelli, "IPv4 Support for Proxy Mobile IPv6," RFC 5844, Tech. Rep., 2010.

[8] B. Carpenter, "Internet Transparency," RFC 2775, Tech. Rep., 2000.

[9] D. Alderson, L. Li, W. Willinger, and J. C. Doyle, "Understanding Internet Topology: Principles, Models, and Validation," IEEE/ACM Trans. on Networking, vol. 13, no. 6, pp. 1205-1218, Dec., 2005.

[10] J. C. Doyle, D. L. Alderson, L. Li, S. Low, M. Roughan, S. Shalunov, R. Tanaka, and W. Willinger, "The "Robust yet Fragile" Nature of the Internet," Proc. of the National Academy of Sciences of the United States of America, vol. 102, no. 41, Oct., 2005.

[11] P. Jokela, A. Zahemszky, C. Esteve Rothenberg, S. Arianfar and P. Nikander, "Lipsin: line speed publish/subscribe internetworking," ACM SIGCOMM Computer Communication Review, vol. 39, no. 4, pp. 195-206, Aug., 2009.

[12] M. J. Reed, M. Al-Naday, N. Thomos, D. Trossen, G. Petropoulos, and S. Spirou, "Stateless multicast switching in software defined networks," in proc. of IEEE Int. Conf. on Communications, ICC'16, Kuala Lumpur, Malaysia, May, 2016.

[13] G. Shepherd and A. Dolganow, "Bit Indexed Explicit Replication (BIER) Problem Statement," Internet-Draft draft-ietf-bier-problemstatement-00, 2016

[14] G. Xylomenos, C. N. Ververidis, V. A. Siris, N. Fotiou, C. Tsilopoulos, X. Vasilakos, K. V. Katsaros, and G. C. Polyzos, "A Survey of Information-Centric Networking Research," IEEE Communications Surveys \& Tutorials, vol. 16, no. 2, pp. 1024-1049, May, 2014.

[15] "PURSUIT project." [Online]. Available: http://www.fp7pursuit.eu

[16] D. Trossen, M. J. Reed, J. Riihijärvi, M. Georgiades, N. Fotiou, and G. Xylomenos, "IP over ICN-The better IP?" in proc. of IEEE European Conf. on Networks and Communications, EuCNC'15, Paris, France, July, 2015.

[17] D. Wall, Managing and securing a Cisco structured wireless-aware network. Syngress, 2004

[18] H. Modares, A. Moravejosharieh, J. Lloret, and R. B. Salleh, "A survey on proxy mobile ipv6 handover," IEEE Systems Journal, vol. 10, no. 1, pp. 208-217, 2016.

[19] D. Trossen and G. Parisis, "Designing and Realizing an Information-Centric Internet," IEEE Communications Magazine, vol. 50, no. 7, pp. 60-67, Jul., 2012.

[20] M. J. Reed, "Traffic Engineering for Information-Centric Networks," in proc. of IEEE Int. Conf. on Communications, ICC'12, Ottawa, Canada, June, 2012.

[21] M. F. Al-Naday, M. J. Reed, D. Trossen, and K. Yang, "Information resilience: source recovery in an information-centric network," IEEE Network, vol. 28, no. 3, pp. 36-42, May 2014.

[22] R. Droms, "Rfc 2131-Dynamic Host Configuration Protocol," Status: DRAFT STANDARD, vol. 3, no. 1, 1997.

[23] T. Choi, L. Kim, J. Nah, and J. Song, "Combinatorial Mobile IP: a New Efficient Mobility Management using Minimized Paging and Local Registration in Mobile IP Environments," Springer Wireless Networks, vol. 10, no. 3, pp. 311-321, May, 2004.

[24] L. Kleinrock, Queueing systems, volume I: theory. Wiley Interscience, 1975

[25] Z. Yan, J.-H. Lee, and Y. Tian, "Localized Paging Scheme in PMIPv6," in proc. of IEEE Int. Conf. on Innovative Mobile and Internet Services in Ubiquitous Computing, IMIS'13, Taichung, Taiwan, July, 2013.

[26] J.-H. Lee and T.-M. Chung, "How Much do we Gain by Introducing Route Optimization in Proxy Mobile IPv6 Networks?" Springer Annals of Telecommunications-Annales des téléCommunications, vol. 65, no. 5-6, pp. 233-246, Jun., 2010.

[27] "Vehicular mobility trace of the city of cologne, germany." [Online]. Available: http:// kolntrace.project.citi-lab.fr/

[28] "Openstreetmap." [Online]. Available: http://www.openstreetmap.org.

[29] "Simulation of urban mobility." [Online]. Available: http://sumo.sourceforge.net.

[30] C. Varschen and P. Wagner, "Mikroskopische modellierung der personenverkehrsnachfrage auf basis von zeitverwendungstage- büchern," Integrierte Mikro-Simulation von Raum-und Verkehrsentwicklung. Theorie, Konzepte, Modelle, Praxis, vol. 81, pp. 63-69, 2006.

[31] K. Blanke, M. Ehling, and N. Schwarz, Zeit im Blickfeld: Ergebnisse einer repräsentativen Zeitbudgeterhebung. Kohlhammer, 1996, vol. 121.

[32] C. Gawron, "An iterative algorithm to determine the dynamic user equilibrium in a traffic simulation model," International Journal of Modern Physics C, vol. 9, no. 03, pp. 393-407, 1998.

[33] A. Lucent, "The LTE Network Architecture: A Comprehensive Tutorial," Strategic Whitepaper, 2009.

[34] K. Taniuchi, Y. Ohba, V. Fajardo, S. Das, M. Tauil, Y.-H. Cheng A. Dutta, D. Baker, M. Yajnik, and D. Famolari, "IEEE 802.21: Media Independent Handover: Features, Applicability, and Realization," IEEE Comms Magazine, vol. 47, no. 1, pp. 112-120, Jan., 2009.

[35] D. Liu and P. Seite, "Distributed Mobility Management: Current Practices and Gap Analysis," RFC 7429, Tech. Rep., 2015.

[36] C. J. Bernardos, A. De la Oliva, and F. Giust, "A PMIPv6-Based Solution for Distributed Mobility Management," Internet-Draft, 2016.

[37] P. S. Kim, M. S. Jang, and E. H. Lee, "An IEEE $802.21 \mathrm{MIH}$ Functionality Assisted Proxy Mobile IPv6 for Reducing Handover Latency and Signaling Cost," in proc. of IEEE Int. Conf. on Information Technology: New Generations, ITNG'13, Las Vegas, USA, Apr., 2013.

[38] Y. Dai, F. Li, H. Li, and Q. Wu, "A core-stateless ip mobility management scheme based on openflow protocol," in Proc. of IEEE Conf. on Wireless Communications and Mobile Computing IWCMC'16, Paphos, Cyprus, Sep., 2016.

[39] S. M. Raza, D. S. Kim, D. Shin, and H. Choo, "Leveraging proxy mobile ipv6 with sdn," Journal of Communications and Networks, vol. 18, no. 3, pp. 460-475, June, 2016.

[40] M. Dong, H. Li, K. Ota, and J. Xiao, "Rule caching in sdn-enabled mobile access networks," IEEE Network, vol. 29, no. 4, pp. 40-45, July, 2015.

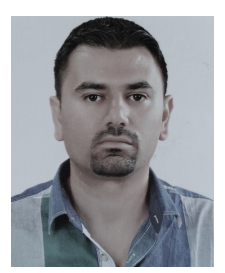

Mohammed Al-Khalidi received his B.Sc. and M.Sc. degrees in Computer and Software Engineering, in 2004 and 2013 respectively. He is currently working towards a Ph.D. degree in Electronic Systems Engineering and also a research officer at the University of Essex, UK. Main research interests are in Network Mobility Management, Information Centric Networks, Network Coding and Internet of Things.

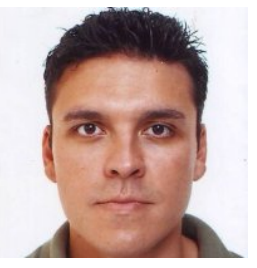

Nikolaos Thomos (S'02-M'06-SM'16) is an associate professor at the University of Essex, UK. He received the Diploma and Ph.D. degrees from Aristotle University of Thessaloniki, Greece in 2000 and 2005 respectively. His research interests include network coding, multimedia communications, information-centric networking, networking, joint source and channe coding, device-to-device communication, and signal processing.

Martin Reed received his Ph.D. from the University of Essex, UK, in 1998. He is currently a Senior Lecture at the University of Essex. His research interests include network control planes, information centric networking, network security and multimedia networking. He has been involved in a number of EPSRC, EU and industrial projects in these areas and has held a Research Fellowship at BT.

Mays Al-Naday is a senior research officer at the University of Essex. She received her PhD degree from the University of Essex in 2015 . During her PhD, she joined the Network Convergence Laboratory and worked as a researcher in number of national and European projects. Her current research interests involve future Internet architectures, 5G networks, Information-centric Networks and multilayer network architectures. 


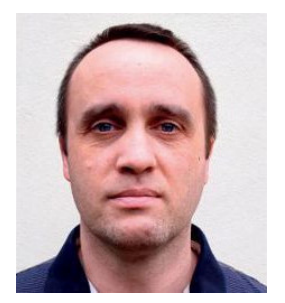

Dirk Trossen is a Senior Principal Engineer at InterDigital Europe. His main responsibility lies in establishing the European presence of InterDigital through engagements within the EU-funded Horizon 2020 work programme as well as within UK-funded efforts. Dirk has more than 15 years of experience in network architectures, services and wireless technology. His main contributions can be found in the area of inter-domain networking as well as seamless handovers and new service concepts for operators. He holds a Ph.D. degree in Computer Science from Technical University of Aachen, Germany. 